\title{
Preorientations of the derived motivic multiplicative group
}

\author{
JENS HORNBOSTEL
}

\begin{abstract}
We establish several new model structures and Quillen adjunctions both in the classical and in the motivic case for algebras over operads and for modules over strictly commutative ring spectra. As an application, we provide a proof in the language of model categories and symmetric spectra of Lurie's Theorem that topological complex $K$-theory represents orientations of the derived multiplicative group. Then we generalize this result to the motivic situation.
\end{abstract}

55P42; 18D50, 19D99, 14F42

\section{Introduction}

In this article, we establish several new model structures and Quillen adjunctions in the motivic setting and study their basic properties. In particular, we establish stable positive model structures for algebras over operads in motivic symmetric spectra. Moreover, we show that both the flat and the projective stable positive model structures on motivic symmetric spectra satisfy the Goerss-Hopkins axioms, and that the flat variant lifts to a model structure on strictly commutative ring spectra which satisfies the Toën-Vezzosi axioms. In short, we make a first step towards a motivic version of derived algebraic geometry.

After the invention of several strict monoidal model categories underlying the stable homotopy category (see Elmendorf, Kriz, Mandell and May [19], Hovey, Shipley and Smith [35]), the subsequent study of commutative algebra of strictly commutative ring spectra has drawn a lot of attention in recent years. Gluing these "derived" commutative ring objects together leads to one of the possible frameworks for derived algebraic geometry, with classical algebraic geometry embedded via the Eilenberg-Mac Lane functor. Derived algebraic geometry through commutative ring spectra has become even more popular when Jacob Lurie gave a conceptual definition of tmf (that is topological modular forms) as the solution of a moduli problem in derived algebraic geometry, as opposed to the handicraft construction of Goerss-Hopkins-Miller; see Behrens [6]. More precisely, Lurie constructs tmf as the global sections of a sheaf of $E_{\infty}$-ring spectra classifying oriented derived elliptic curves. He has sketched the proof of this theorem in [42], and has lectured about various parts of it at various places. 
According to him, the best language to state and prove the theorem is the one of infinity categories rather than the one of model categories. Here infinity categories really mean quasicategories, also known as weak Kan complexes, as first invented by Boardman and Vogt [13] and recently studied in great detail by Lurie, Joyal and others. The interested reader should consult [41; 43], as well as Bergner [9] for a comparison with other approaches to infinity categories. We expect that Lurie will publish a detailed proof of his theorem in this language in the near future, the books [41] and [43] containing already most of the necessary language and machinery.

The above description of tmf (corresponding to height two and the second chromatic layer) has an analog in height 1 which is much easier to state and to prove, and is also due to Lurie [42, Section 3]. Namely, real topological $K$-theory $K O$ classifies oriented derived multiplicative groups. The key step for proving this is to show that the suspension spectrum of $\mathrm{CP}^{\infty}$ classifies preorientations of the derived multiplicative group. Here the derived multiplicative group is by definition $\mathbf{G}_{m}:=\operatorname{Spec}\left(\Sigma^{\infty} \mathbb{Z}_{+}\right)$, the name being justified by classical algebraic geometry over a base field $k$, where the multiplicative group is $\operatorname{Spec}(k[\mathbb{Z}])$. As usual, the object $\left.\operatorname{Rmap}_{\operatorname{AbMon}\left(\operatorname{Sp}^{\Sigma}\right)}\left(\Sigma^{\infty} \mathbb{Z}_{+},-\right)\right)$ it represents via the derived version of the Yoneda embedding will still be called the multiplicative group. (In the present article, all arguments take place in the affine derived setting, so there is no need to write Spec and to reverse the order of the arrows everywhere.) We will provide a proof of this result in the language of model categories and symmetric spectra. For this we tacitly assume $\mathrm{CP}^{\infty}$ (compare [42]) that the topological monoid $\mathrm{CP}^{\infty}$ has been replaced by a homotopy equivalent model that is actually a topological resp. simplicial group. Then the result reads as follows in general, the special case $N=\mathrm{CP}^{\infty}$ being the one discussed above.

Theorem 1.1 (Lurie) For any abelian monoid $A$ in symmetric spectra $\mathrm{Sp}^{\Sigma}$ (based on simplicial sets) and any simplicial abelian group $N$, we have a natural isomorphism of abelian groups

$$
\begin{aligned}
\operatorname{Hom}_{\mathrm{Ho}\left(\operatorname{AbMon}\left(\mathrm{Sp}^{\Sigma}\right)\right)}\left(\Sigma^{\infty}\right. & \left.N_{+}, A\right) \\
& \simeq \operatorname{Hom}_{\operatorname{Ho}\left(\operatorname{AbMon}\left(\Delta^{\mathrm{op} S e t s}\right)\right)}\left(N, \operatorname{Rmap}_{\mathrm{AbMon}\left(\mathrm{Sp}^{\Sigma}\right)}\left(\Sigma^{\infty} \mathbb{Z}_{+}, A\right)\right) \\
& =\operatorname{Hom}_{\mathrm{Ho}\left(\operatorname{AbMon}\left(\Delta^{\mathrm{op} S e t s}\right)\right)}\left(N, \mathbf{G}_{m}(A)\right) .
\end{aligned}
$$

Here Ho(-) denotes the homotopy category, Rmap means the derived mapping space and the weak equivalences between abelian monoids are always the underlying ones, forgetting the abelian monoid structure. The model structures involved in this statement are discussed in detail in Section 3. Beware that in general the category of abelian monoids in a homotopy category of a monoidal model category is different from the 
homotopy category of abelian monoids in the monoidal model category, the monoidal model category $\left(\Delta^{\mathrm{op}}\right.$ Sets, $\left.\times\right)$ and the abelian monoid $Q S^{0}$ in $\mathrm{Ho}\left(\Delta^{\mathrm{op}}\right.$ Sets $)$ being the most prominent example.

We will prove Theorem 1.1 in Section 4, and indeed a much more general version (see below). Using a theorem of Snaith [64], Lurie's definition of an orientation and his above theorem then imply that complex $K$-theory represents orientations of the standard derived multiplicative group, and then further that real $K$-theory represents oriented derived multiplicative groups in general. We refer the reader to Section 5 for further details.

The above theorem can be generalized to motivic symmetric spectra $\operatorname{Sp}^{\Sigma, T}(\mathcal{M})$ on the smooth Nisnevich site $\mathcal{M}=(\mathrm{Sm} / S)_{\text {Nis }}$ with $S$ an arbitrary Noetherian base scheme as follows, everything equipped with appropriate motivic (that is $\mathbf{A}^{1}$-local) model structures as discussed in Section 3, and the motivic derived multiplicative group $\mathbf{G}_{m}^{\text {mot }}$ defined using the suspension spectrum with respect to a given motivic circle $T$, that is represented by $\Sigma_{T}^{\infty} \mathbb{Z}_{+}$. (As the notation suggests, we are mainly interested in the circle provided by $\mathbf{A}^{1} /(\mathbf{A}-0)$ resp. the weakly equivalent $T=\mathbf{P}^{1}$ pointed at infinity.)

Theorem 1.2 Let $\mathcal{M}=(S m / S)_{\mathrm{Nis}}$ and $T=S^{1}$ or $T=\mathbf{P}^{1}$. Then for any abelian monoid $A$ in motivic symmetric $T$-spectra $\operatorname{Sp}^{\Sigma, T}(\mathcal{M})$ and any abelian group $N$ in the category $\Delta^{\mathrm{op}} \operatorname{PrShv}(\mathcal{M})$ of simplicial presheaves on $\mathcal{M}$, we have a natural isomorphism of abelian groups

$$
\begin{aligned}
& \operatorname{Hom}_{\mathrm{Ho}\left(\operatorname{AbMon}\left(\mathrm{Sp}^{\Sigma, T}(\mathcal{M})\right)\right)}\left(\Sigma_{T}^{\infty} N_{+}, A\right)
\end{aligned}
$$

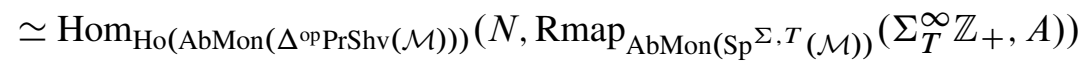

$$
\begin{aligned}
& =\operatorname{Hom}_{H o}\left(\operatorname{AbMon}\left(\Delta^{\operatorname{opPrShv}}(\mathcal{M})\right)\right)\left(N, \mathbf{G}_{m}(A)\right) \text {. }
\end{aligned}
$$

This is a rather straightforward application of the main technical results of this article. Applying it to $T=\mathbf{P}^{1}$ pointed at $\infty$ and to $N=\mathbf{P}^{\infty}$, which is not a variety but still a simplicial presheaf, and using the recently established motivic version of Snaith's Theorem (see Gepner and Snaith [22] and Spitzweck and Østvær [66], it will imply that algebraic $K$-theory represents motivic orientations of the derived motivic multiplicative group, provided one works with the correct motivic generalizations of the concept of derived algebraic groups and of orientations. Again, we refer to Section 5 for details, as well as for possible connections to hermitian $K$-theory which in many ways is the motivic analog of topological real $K$-theory.

One of the many motivations of this articleis that the generalizations of the language of derived algebraic geometry from classical to motivic spectra should ultimately lead to 
a definition of a motivic version of $t m f$, generalizing the above Theorem 1.1 of Lurie about height 2 to the motivic setup as well. We will not pursue this in the present article. (Note that the recent article by Naumann, Spitzweck and Østvær [49] allows to define motivic elliptic cohomology theories and motivic elliptic ring spectra via motivic Landweber exactness.) However, concerning motivic derived algebraic geometry, we wish to point out three interesting applications of the results of this article. First, motivic symmetric $\mathbf{P}^{1}$-spectra equipped with a suitable positive model structure satisfy the axioms of a $H A$-context of Toën and Vezzosi [67], so their machinery applies to this example. See Section 3.4 for a detailed discussion. Second, we have suitable model structures on algebras over stable motivic operads (see Theorems 3.6 and 3.10).

Theorem 1.3 Let $T=S^{1}$ or $T=\mathbf{P}^{1}$, and let $\mathcal{O}$ be any operad in $\operatorname{Sp}^{\Sigma, T}(\mathcal{M})$, and consider $\mathrm{Sp}^{\Sigma, T}(\mathcal{M})$ with the positive flat stable model structure established in Theorem 3.4. Then the forgetful functor from $\mathcal{O}$-alg to $\mathrm{Sp}^{\Sigma, T}(\mathcal{M})$ creates a model structure on $\mathcal{O}_{-}$alg in the sense of Schwede and Shipley [61, Lemma 2.3]. Moreover, any Quillen equivalence of operads leads to Quillen equivalent categories of algebras.

Third, the motivic analogue of the axioms of Goerss and Hopkins [23] is also satisfied (see Theorem 3.15). I understand that this third application was established simultaneously and independently by Paul Arne Østvær, who wants to use it for doing motivic obstruction theory. Both applications are presented in Section 3.

We pause to make some comments concerning the proof of Theorem 1.2, which is given in Section 4 and uses the results established in Section 3. First, one should notice that the theorem is about $T$-spectra, but even for $T=\mathbf{P}^{1}$ the proof involves motivic $S^{1}$-spectra as well. This is mainly due to the fact that at some point one needs a motivic version of the recognition principle which relates $E_{\infty}$-spaces to connective $S^{1}$-spectra. The classical recognition principle is a statement about $S^{1}$-deloopings, and our generalization of it to motivic $S^{1}$-spectra is sufficient for our purposes. Finding a recognition principle for motivic $\mathbf{P}^{1}$-spectra, that is a motivic operad encoding $\mathbf{G}_{m^{-}}$ or $\mathbf{P}^{1}$-deloopings, remains one of the main open problems in motivic homotopy theory, as already pointed out by Voevodsky in [69, introduction]. To show that a motivic version of the recognition principle with respect to $S^{1}$ holds, a previous version of this articleinvoked the beautiful $\mathbf{A}^{1}$-connectivity theorem of Fabien Morel [47], which is known only over a field. (It seems to be an open question if Morel's Theorem also holds for 1-dimensional base schemes. For 2-dimensional base schemes, there is a counterexample due to Ayoub.) However, we later realized that the proof does not really require this result and hence holds for more general base schemes.

At first glance, it might also be surprising that we need $E_{\infty}$-structures to prove a theorem about strictly commutative monoids in strictly monoidal model categories. 
This is essentially a consequence of the Lewis paradoxon, as explained at the end of Section 2. If one is only interested in strict adjunctions and willing to ignore all derived information, and in particular to sacrifice homotopy invariance of the statement, then there is a much easier proof not using operads, which we present in Section 2. At the beginning of Section 2, we fix some notation which will be used throughout this article.

Several results of this article may be generalized to other left Bousfield localizations of simplicial presheaves on a site (or even on other diagram categories satisfying some cardinality conditions), and similarly for the stable case. See the Remark after Theorem 3.6 for a more precise statement in this direction.

The referee points out that it should be possible to prove not only Theorem 1.1, but also Theorem 1.2 using infinity-category techniques. This would require eg to work with the infinity category of $\mathbf{A}^{1}$-local sheaves in some appropriate infinity-category sense. As I am not an expert in that field, and as the focus of this article is on the relevant model structures for motivic model rather than infinity categories, I will not try to speculate on the details of this presumably much simpler proof here. The recent preprint [55] provides many of the relevant results concerning the infinity theoretic description of the motivic categories.

This work started as a joint project with Niko Naumann, and was presented as such on a conference in Münster in July 2009. I am indebted to him for the many discussions we had on the topics of this article. Some parts of the work presented here have been obtained in joint work or are at least influenced by these discussions, and I thank him for allowing me to include these parts here. Moreover, I wish to thank Stefan Schwede, John Harper and Benoit Fresse for discussions and explanations about certain points in their works concerning model structures for classical symmetric spectra, operads over them and $E_{\infty}$-operads, respectively, as well as Jacob Lurie for some explanations about [42] and Pablo Pelaez for discussions related to [52; 47].

\section{The nonderived situation: preorientations which are not homotopy invariant}

The main goal of this section is to establish the following theorem, which is a nonderived analogue of Theorems 1.1 and 1.2. All the objects involved are defined below, and the proof of the theorem is given by suitably combining the lemmata in this section.

Theorem 2.1 Let $\mathcal{C}$ be an essentially small a category, let $M$ be an abelian group object in $\Delta^{\mathrm{op}} \operatorname{PrShv}(\mathcal{C})$ and $A$ an abelian monoid object in $\operatorname{Sp}^{\Sigma, T}(\mathcal{C})$. Then we have a natural adjunction isomorphism of simplicial sets

$$
\operatorname{map}_{\mathrm{AbMonSp}^{\Sigma, T}(\mathcal{C})}(S[M], A) \cong \operatorname{map}_{\mathrm{AbMon} \Delta^{\operatorname{opPrShv}(\mathcal{C})}}\left(M, \mathbf{G}_{m}^{\prime}(A)\right) .
$$


We now introduce some notation. For any monoidal category $\mathcal{D}=(\mathcal{D}, \otimes)$, we denote the category of monoid objects in $\mathcal{D}$ by $\operatorname{Mon} \mathcal{D}$, and the one of abelian monoids by $\mathrm{AbMon} \mathcal{D}$. When talking about abelian monoids, we assume moreover that the monoidal categories and functors involved are symmetric. In our applications, $\otimes$ will be either the cartesian product $\times$ or some smash product $\wedge$. All monoids are assumed to be associative, but not necessarily unital. We refer the reader to Mac Lane [44] for precise definitions of monoidal categories, (strong) monoidal functors etc.

We fix a category $\mathcal{C}$ from now on. In applications $\mathcal{C}$ will be a site, more specifically either the trivial site or the site $(S m / k)_{\text {Nis }}$ of smooth $k$-schemes, $k$ a field, with the Nisnevich topology. We denote the category of simplicial presheaves on $\mathcal{C}$ by $\Delta^{\mathrm{op}} \operatorname{PrShv}(\mathcal{C})$. For a given simplicial presheaf $T$, we denote the category of presheaves of symmetric $T$-spectra on $\mathcal{C}$ by $\mathrm{Sp}^{\Sigma, T}(\mathcal{C})$. Model structures on these categories are discussed in Morel and Voevodsky [48], Jardine [37], Hovey [34] and elsewhere, but we will not need them in this subsection. One might wish to call commutative monoids in $\operatorname{Sp}^{\Sigma, T}(\mathcal{C})$ "commutative motivic symmetric ring spectra" in case $\mathcal{C}=(\mathrm{Sm} / \mathrm{k})_{\text {Nis }}$, resp. "commutative symmetric ring spectra" in case $\mathcal{C}=p t$.

Adding a disjoint base point is denoted by $(\cdot)_{+}$and yields a left adjoint to the functor $F$ forgetting the base point in various situations. For a simplicial presheaf $X$, we set $S[X]:=\Sigma_{T}^{\infty}\left(X_{+}\right) \in \operatorname{Sp}^{\Sigma, T}(\mathcal{C})$ which is defined objectwise as in [35, Definition 2.2.5] or Schwede [60, Example 1.2.6]. If $X$ is a monoid in the monoidal category $\left(\Delta^{\mathrm{op}} \operatorname{PrShv}(\mathcal{C}), \times\right)$, then $X_{+}$is a monoid in the monoidal category $\left(\Delta^{\mathrm{op}} \operatorname{PrShv}(\mathcal{C})_{\bullet}, \wedge\right)$ of pointed simplicial presheaves and $S[X]$ is a monoid in the monoidal category $\left(\operatorname{Sp}^{\Sigma, T}(\mathcal{C}), \wedge\right)$ of presheaves of symmetric $T$-spectra; see Lemma 2.3 below. We denote the functor sending a presheaf of symmetric $T$-spectra to the simplicial presheaf sitting in degree 0 by $E v_{0}$.

For simplicial presheaves $\mathcal{F}$ and $\mathcal{G}$ we have a $\operatorname{simplicial}$ set $\operatorname{map}_{\Delta^{\mathrm{op} P r S h v}(\mathcal{C})}(\mathcal{F}, \mathcal{G})$ given by $\operatorname{map}_{\Delta^{\text {opPrShv }}(\mathcal{C})}(\mathcal{F}, \mathcal{G})_{n}:=\operatorname{Hom}_{\Delta^{\text {opPrShv }}(\mathcal{C})}\left(\mathcal{F} \times \Delta^{n}, \mathcal{G}\right)$. We define the simplicial presheaf $\operatorname{map}_{\Delta^{\text {opPrShv }}(\mathcal{C})}(\mathcal{F}, \mathcal{G})$ by $\operatorname{map}_{\Delta^{\operatorname{opPrShv}}(\mathcal{C})}(\mathcal{F}, \mathcal{G})(c)=\operatorname{map}_{\Delta^{o p}(\mathcal{C} / c)}(\mathcal{F}|c, \mathcal{G}| c)$ where $F \mid c$ denotes the restriction of the (simplicial) presheaf $\mathcal{F}$ to the category $\mathcal{C} / c$ of objects in $\mathcal{C}$ lying over $c$. For presheaves of symmetric $T$-spectra, we define the simplicial sets $\operatorname{map}_{\mathrm{Sp}^{\Sigma, T}(\mathcal{C})}$ and simplicial presheaves $\operatorname{map}_{\mathrm{Sp}^{\Sigma, T}(\mathcal{C})}$ in a similar way. Forgetting about simplicial enrichments, we write Hom for the presheaf version of Hom.

Finally, we define the (nonderived) multiplicative group as follows, the derived version of the introduction being the one using the derived mapping space Rmap instead.

Definition 2.2 The nonderived multiplicative group is the functor

$$
\mathbf{G}_{m}^{\prime}: \operatorname{AbMonSp}^{\Sigma, T}(\mathcal{C}) \rightarrow \operatorname{AbMon} \Delta^{\mathrm{op}} \operatorname{PrShv}(\mathcal{C})
$$


given by

$$
\mathbf{G}_{m}^{\prime}(A):=\operatorname{map}_{\mathrm{AbMonSp}^{\Sigma, T}(\mathcal{C})}(S[\mathbb{Z}], A),
$$

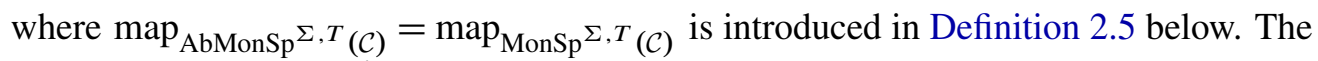
monoid structure on $\mathbf{G}_{m}^{\prime}(A)$ is induced by the comonoid structure on $S[\mathbb{Z}]$, the latter lifting the one on $\mathbb{Z}[\mathbb{Z}]$ corresponding to the multiplicative group in algebraic geometry over $\operatorname{Spec}(\mathbb{Z})$.

We define monoidal and strict monoidal functors between monoidal categories and monoidal transformations between (strong) monoidal functors as in [44, Chapter XI]. An adjunction between monoidal categories is called a monoidal adjunction if the unit and the counit are monoidal transformations. One easily checks that a monoidal functor sends monoids to monoids. The following lemma is well known.

\section{Lemma 2.3 (i) We have a monoidal adjunction}

$$
(\cdot)_{+}: \Delta^{\mathrm{op}} \operatorname{PrShv}(\mathcal{C}) \rightleftarrows \Delta^{\mathrm{op}} \operatorname{PrShv}(\mathcal{C})_{\bullet}: F,
$$

where $(\cdot)_{+}$is strong monoidal and the forgetful functor $F$ is monoidal. Consequently, we have isomorphisms

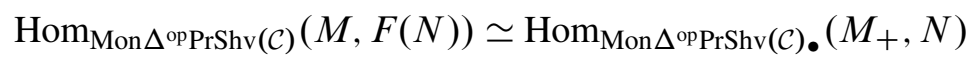

for any unpointed monoid $M \in \operatorname{Mon} \Delta^{\mathrm{op}} \operatorname{PrShv}(\mathcal{C})$ and any pointed monoid $N \in \operatorname{Mon} \Delta^{\mathrm{op}} \operatorname{PrShv}(\mathcal{C})$.

(ii) We have a monoidal adjunction

$$
\Sigma_{T}^{\infty}: \Delta^{\mathrm{op}} \operatorname{PrShv}(\mathcal{C}) \bullet \rightleftarrows \operatorname{Sp}^{\Sigma, T}(\mathcal{C}): E v_{0},
$$

where both functors are strong monoidal. Consequently, we have isomorphisms

$$
\operatorname{Hom}_{\operatorname{Mon} \Delta} \operatorname{opPrShv}(\mathcal{C}) \bullet\left(A, E v_{0}(B)\right) \simeq \operatorname{Hom}_{\operatorname{MonSp}^{\Sigma, T}(\mathcal{C})}\left(\Sigma_{T}^{\infty} A, B\right)
$$

for any monoid $A$ in $\Delta^{\mathrm{op}} \operatorname{PrShv}(\mathcal{C}) \bullet$ and any monoid $B$ in $\operatorname{Sp}^{\Sigma, T}(\mathcal{C})$.

(iii) We have a monoidal adjunction

$$
S[\cdot]: \Delta^{\mathrm{op}} \operatorname{PrShv}(\mathcal{C}) \rightleftarrows \mathrm{Sp}^{\Sigma, T}(\mathcal{C}): F \circ E v_{0},
$$

where $S[\cdot]$ is strong monoidal and $F \circ E v_{0}$ is monoidal. Consequently, we have isomorphisms

$$
\operatorname{Hom}_{M o n} \Delta^{\operatorname{op} \operatorname{PrShv}(\mathcal{C})}\left(M, F \circ E v_{0}(B)\right) \simeq \operatorname{Hom}_{\operatorname{MonSp}^{\Sigma, T}(\mathcal{C})}(S[M], B)
$$

for any monoid $M$ in $\Delta^{\mathrm{op}} \operatorname{PrShv}(\mathcal{C})$ and any monoid $B$ in $\operatorname{Sp}^{\Sigma, T}(\mathcal{C})$. 
Proof Part (i) is straightforward. The morphisms of [44, XI.2.(1), (2)] for the monoidal functor $F$ are given by the quotient map $X \times Y \rightarrow X \wedge Y$ and by $p t \rightarrow(p t)_{+}=S^{0}$. The final statement follows from the obvious remark that a monoidal adjunction induces an adjunction between categories of monoids.

Part (ii) is checked objectwise, using [35, Definition 2.2.5, Proposition 2.2.6.1 and Definition 2.1.3] or the corresponding results in [60] and then proceeding similar to part (i).

Part (iii) is obtained by composing (i) and (ii).

Definition 2.4 Let $(\mathcal{D}, \otimes)$ be a monoidal category such that the underlying category is enriched over simplicial sets. We say that $(\mathcal{D}, \otimes)$ satisfies $(\mathrm{MS})$ if there is a natural transformation of simplicial sets $\tau_{x, y, z, w}: \operatorname{map}(x, y) \times \operatorname{map}(z, w) \rightarrow \operatorname{map}(x \otimes z, y \otimes w)$ which on map $(,)_{0}=$ Hom coincides with the transformation sending $(f, g)$ to $f \otimes g$, and we say that $(\mathcal{D}, \otimes, \tau)$ is a simplicial monoidal category.

The property (MS) may be rephrased by saying that $\otimes$ is enriched in simplicial sets.

Definition 2.5 Let $(\mathcal{D}, \otimes, \tau)$ be a simplicial monoidal category. Then for any monoids $\left(x, m_{x}\right)$ and $\left(y, m_{y}\right)$ in $\mathcal{D}$, we define $\operatorname{map}_{\text {Mon }}(x, y) \subset \operatorname{map}(x, y)$ to be the equalizer of $\operatorname{map}\left(m_{x}, y\right): \operatorname{map}(x, y) \rightarrow \operatorname{map}(x \otimes x, y)$ and $\operatorname{map}\left(x \otimes x, m_{y}\right) \circ \tau_{x, y, x, y} \circ$ $\Delta: \operatorname{map}(x, y) \rightarrow \operatorname{map}(x \otimes x, y)$, where $\tau_{x, y, x, y}$ is as in Definition 2.4. If we have $\mathcal{D}=\Delta^{\mathrm{op}} \operatorname{PrShv}(\mathcal{C}), \mathcal{D}=\Delta^{\mathrm{op}} \operatorname{PrShv}(\mathcal{C}) \bullet$ or $\mathcal{D}=\operatorname{Sp}^{\Sigma, T}(\mathcal{C})$, then we denote the presheaf version of map $_{M o n}$ by mapon $_{M o n}$, and the one of Hom Mon $_{\text {bo }}$ Hom $_{M o n}$.

Lemma 2.6 Lemma 2.3 above remains true when replacing Hom by Hom or by map everywhere.

Proof The isomorphisms for Hom $\mathrm{Mon}_{\text {formally imply those for Hom }}$ Mon . The claim

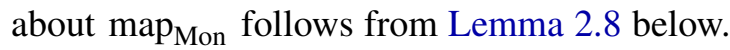

Definition 2.7 Let $\left(\mathcal{C}, \otimes_{\mathcal{C}}, \tau\right)$ and $\left(\mathcal{D}, \otimes_{\mathcal{D}}, \tau\right)$ be simplicial monoidal categories as in Definition 2.4. Let $F: \mathcal{C} \rightarrow \mathcal{D}$ be a functor of simplicial categories such that the underlying functor of categories is a monoidal functor with structure maps $F_{2}: F(x) \otimes_{\mathcal{D}} F(y) \rightarrow F\left(x \otimes_{\mathcal{C}} y\right)$ and $F_{0}: 1_{\mathcal{D}} \rightarrow F\left(1_{\mathcal{C}}\right)$. We say that $F$ is a simplicial monoidal functor if for any objects $x, y$ of $\mathcal{C}$ the diagram

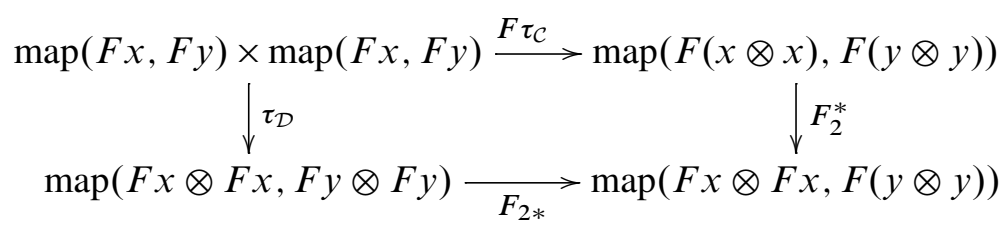

commutes. 
Lemma 2.8 (i) Assume that $\left(\mathcal{D}_{i}, \otimes_{i}, \tau_{i}\right), i=1,2$ are simplicial monoidal categories and that

$$
\alpha: \mathcal{D}_{1} \leftrightarrows \mathcal{D}_{2}: \beta
$$

is a simplicial monoidal adjunction, ie $\alpha$ and $\beta$ are simplicial monoidal functors and there is a monoidal adjunction between the underlying monoidal functors. Then for $\left(x_{i}, m_{i}\right)$ monoids in $\mathcal{D}_{i}, i=1,2$, we have an isomorphism

$$
\operatorname{map}_{\mathrm{Mon} \mathcal{D}_{1}}\left(x_{1}, \beta x_{2}\right) \simeq \operatorname{map}_{\mathrm{Mon}_{2}}\left(\alpha x_{1}, x_{2}\right)
$$

of simplicial sets, and similarly for map.

(ii) The monoidal categories $\left(\Delta^{\mathrm{op}} \operatorname{PrShv}(\mathcal{C}), \times\right),\left(\Delta^{\mathrm{op}} \operatorname{PrShv}(\mathcal{C}) \bullet, \wedge\right)$ and $\left(\operatorname{Sp}^{\Sigma, T}(\mathcal{C}), \wedge\right)$ are enriched over simplicial sets as categories and satisfy $(M S)$ with respect to the obvious choices of $\tau$, hence are simplicial monoidal categories.

(iii) The monoidal adjunctions of Lemma 2.3 are simplicial.

Proof The proof of (i) is a little long but straightforward. In part (ii), for constructing the transformations $\tau$ required in (MS) one uses the diagonal $\Delta_{+}^{n} \rightarrow \Delta_{+}^{n} \wedge \Delta_{+}^{n}$, the twist and that for any simplicial presheaf $K$ (in particular for $K=\Delta^{n}$ ) and any $X \in \mathrm{Sp}^{\Sigma, T}(\mathcal{C})$ one has $K \wedge X=\left(\Sigma_{T}^{\infty} K\right) \wedge X$ which can be shown objectwise using the results of [60, Chapter I]. For part (iii), use that all mapping spaces involved are defined using the standard cosimplicial object, and the composition is defined using the diagonal on it.

Observe that for any monoidal category $\mathcal{C}, \operatorname{Hom}_{\operatorname{Mon} \mathcal{C}}(A, B)=\operatorname{Hom}_{\mathrm{AbMonC}}(A, B)$ for any abelian monoid objects $A$ and $B$ in $\mathcal{C}$, and similar for Hom, map and map, eg, both Lemma 2.3 and Lemma 2.9 below restrict to abelian monoids. We now restrict our discussion to unital monoids. For $M \in \operatorname{Mon} \Delta^{\mathrm{op}} \operatorname{PrShv}(\mathcal{C})$, we denote by $M^{\times}$the group object in $\Delta^{\mathrm{op}} \operatorname{PrShv}(\mathcal{C})$ defined by $\left(M^{\times}\right)_{k}=\left(M_{k}\right)^{\times}$, that is taking objectwise the invertible elements in each simplicial degree. These units satisfy the following.

Lemma 2.9 (i) For any $N \in \operatorname{Mon} \Delta^{\operatorname{op}} \operatorname{PrShv}(\mathcal{C})$ a simplicially constant group object and $M \in \operatorname{Mon} \Delta^{\mathrm{op}} \operatorname{PrShv}(\mathcal{C})$, one has an isomorphism

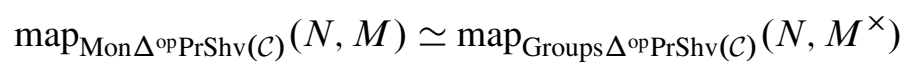

and similar for map. In particular, if $N=\mathbb{Z}$ one has

$$
\operatorname{map}_{M o n \Delta} \operatorname{opPrShv}(\mathcal{C})(\mathbb{Z}, M) \simeq M^{\times} .
$$


(ii) More generally, if $N$ is a group object in $\Delta^{\mathrm{op}} \operatorname{PrShv}(\mathcal{C})$, then one has an isomorphism

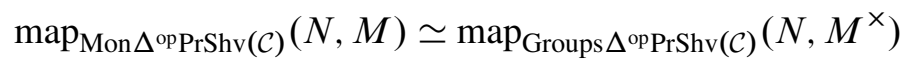

and similarly for map.

Proof For simplicially constant $N$, one has isomorphisms

$$
\begin{aligned}
& \operatorname{map}_{\operatorname{Mon} \Delta{ }^{\mathrm{opPrShv}}(\mathcal{C})}(N, M)_{n} \simeq \operatorname{Hom}_{\operatorname{Mon} \Delta^{\mathrm{opPrShv}}(\mathcal{C})}\left(N, \operatorname{map}_{\Delta^{\mathrm{opPrShv}}(\mathcal{C})}\left(\Delta^{n}, M\right)\right)
\end{aligned}
$$

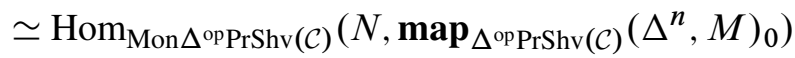

$$
\begin{aligned}
& \simeq \operatorname{Hom}_{\operatorname{Mon} \Delta} \operatorname{opPrShv}(\mathcal{C})\left(N, M_{n}\right) \text {, }
\end{aligned}
$$

where the first isomorphism holds because $(\cdot)_{n}$ commutes with limits. The monoid structure on $\operatorname{map}_{\Delta^{\mathrm{opPrSh}}(\mathcal{C})}\left(\Delta^{n}, M\right)$ is defined by composing $\tau$, the diagonal $\Delta^{n} \rightarrow \Delta^{n} \times \Delta^{n}$ and the monoid structure of $M$. Using these isomorphisms, part (i) about map reduces to the corresponding well-known result for usual monoids, and the result about map follows formally from this by definition. For the claim about $\mathbb{Z}$ use that $\operatorname{map}_{M o n} \Delta^{\operatorname{opPrShv}(\mathcal{C})}(\mathbb{Z}, M)_{n} \simeq \operatorname{Hom}_{\mathrm{Mon}}\left(\mathbb{Z}, \operatorname{map}_{\Delta^{\operatorname{opPrShv}(\mathcal{C})}}\left(\Delta^{n}, M\right)\right)$ and that $\operatorname{Hom}_{M o n}(\mathbb{Z}, M)=M^{\times}$for usual monoids $M$. For (ii), first check

$$
\begin{aligned}
& \operatorname{map}_{\Delta^{\mathrm{opPrShv}}(\mathcal{C})}\left(\Delta^{n} \times \Delta^{k}, \operatorname{map}_{\operatorname{Mon} \Delta^{\mathrm{opPrShv}}(\mathcal{C})}(\mathbb{Z}, M)\right) \\
& \simeq \operatorname{map}_{\operatorname{Mon} \Delta^{\mathrm{oPPrShv}}(\mathcal{C})}\left(\mathbb{Z}, \operatorname{map}_{\Delta^{\mathrm{opPrShv}(\mathcal{C})}}\left(\Delta^{n} \times \Delta^{k}, M\right)\right),
\end{aligned}
$$

as both are subsets of $\operatorname{map}_{\Delta^{\text {opPrShv }}(\mathcal{C})}\left(\Delta^{n} \times \Delta^{k} \times \mathbb{Z}, M\right)$ defined by the same diagrams. As $k$ varies, this implies an isomorphism of simplicial groups

$$
\operatorname{map}_{\Delta^{\mathrm{opPrShv}}(\mathcal{C})}\left(\Delta^{n}, M^{\times}\right) \simeq \operatorname{map}_{\Delta^{\mathrm{opPrSh}}(\mathcal{C})}\left(\Delta^{n}, M\right)^{\times},
$$

where the monoid structure on the right is given by the one on $M$. Applying $\operatorname{Hom}_{\operatorname{Mon} \Delta^{\operatorname{opPrShv}}(\mathcal{C})}(N$,$) and using part (i), one deduces that$

$$
\begin{aligned}
& \operatorname{Hom}_{\operatorname{Mon} \Delta^{\mathrm{opPrShv}}(\mathcal{C})}\left(N, \operatorname{map}_{\Delta^{\mathrm{opPrShv}}(\mathcal{C})}\left(\Delta^{n}, M^{\times}\right)\right) \\
& \simeq \operatorname{Hom}_{\operatorname{Mon} \Delta^{\mathrm{opPrShv}}(\mathcal{C})}\left(N, \operatorname{map}_{\Delta^{\mathrm{opPrShv}}(\mathcal{C})}\left(\Delta^{n}, M\right)^{\times}\right)
\end{aligned}
$$

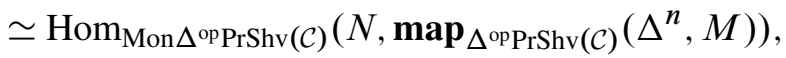

and the claim now follows by varying $n$, using the adjunction between map and $\times$. 
Using the above results, Theorem 2.1 now follows from the following chain of isomorphisms using the amplification of the indicated results provided by Lemma 2.6:

$\operatorname{map}_{\mathrm{MonSp}^{\Sigma, T}(\mathcal{C})}(S[M], A)$

$$
\begin{aligned}
& \stackrel{2.3 \text { (iii) }}{\simeq} \operatorname{map}_{\operatorname{Mon} \Delta \operatorname{opPrShv}(\mathcal{C})}\left(M, F \circ E v_{0}(A)\right) \\
& \stackrel{2.9 \text { (ii) }}{\simeq} \operatorname{map}_{\mathrm{Groups} \Delta} \operatorname{op\operatorname {PrShv}(\mathcal {C})}\left(M,\left(F \circ E v_{0}(A)\right)^{\times}\right)
\end{aligned}
$$

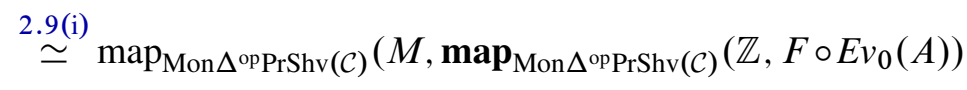

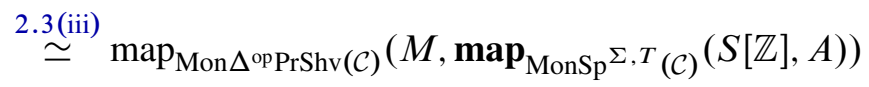

$$
\begin{aligned}
& =\operatorname{map}_{\mathrm{Mon} \Delta{ }^{\mathrm{opPrShv}(\mathcal{C})}}\left(M, \mathbf{G}_{m}^{\prime}(A)\right) \text {. }
\end{aligned}
$$

Recall that we may replace Mon by AbMon everywhere.

Now, what happens if we try to give Theorem 2.1 a homotopy theoretic meaning, equipping everything with suitable model structures? One problem that may arise is the definition of the multiplicative group in Theorems 1.1 and 1.2 using the derived mapping spaces with respect to the chosen model structures. It is not clear if there is a cofibrant replacement of $S[\mathbb{Z}]$ which is also a comonoid, that is an affine derived group scheme. One may try to show that the functor represented by $\mathbf{G}_{m}^{\prime}$ is weakly equivalent to one factoring through simplicial abelian groups. Independently (in fact maybe not completely independently) of this, the main problem seems to be the following. Making our proof homotopy invariant means that all adjunctions involved have to be Quillen, and that will be impossible to achieve. The problem that appears does so already for the trivial category $\mathcal{C}$ with a single object and no nontrivial automorphisms, that is for classical homotopy theory. Consider the adjunction of Lemma 2.3(iii), restricted to abelian monoids. We want the model structure on $\operatorname{AbMon}\left(\Delta^{\mathrm{op}}\right.$ Sets $)$ to be the usual one. For $\mathrm{Sp}^{\Sigma}$, we have essentially two families of model structures, namely the usual ones and the positive ones. If we choose a usual nonpositive stable model structure, then this will not lift to a model structure on $\operatorname{AbMon}\left(\operatorname{Sp}^{\Sigma}\right)$ with weak equivalences and fibrations defined using the forgetful functor to $\mathrm{Sp}^{\Sigma}$ because of the Lewis paradoxon; see eg Mandell, May, Schwede and Shipley [45, Section 14] or [61, Remark 4.5]. The fact that this adjunction is not Quillen for any reasonable model structure on abelian monoids is why we have to work so much more in the next two sections, using motivic versions of $E_{\infty}$-spectra, of the recognition principle, of a theorem of Schwede and Shipley [62] establishing a zigzag of Quillen equivalences between $H \mathbb{Z}$-modules in symmetric spectra and unbounded complexes of abelian groups, etc. This should not be considered as a technical problem about model category theory or symmetric spectra, but as an honest mathematical problem related to the stable homotopy type of the 
sphere spectrum and the content of our main theorem. Therefore it will appear in some way or another in any language one might choose to deal with these questions.

\section{Model structures for algebras over operads in symmetric spectra and applications}

The goal of this section is to show that the category of motivic symmetric spectra as considered by Jardine [37] and Hovey [34] equipped with suitable model structures satisfies all properties necessary for a motivic version of derived algebraic geometry. More precisely, we show (see Section 3.4) that motivic symmetric spectra together with suitable model structures enable us to construct model structures for algebras in motivic symmetric spectra under a given operad, motivic symmetric spectra satisfy the assumptions of [67, Section 1.1] when choosing suitable additional data (except that we do not discuss possible choices of $\mathcal{C}_{0}$ and $\mathcal{A}$ as introduced in [67, 1.1.06, 1.1.0.11] here). They also satisfy a motivic variant of the axioms of [23, 1.1 and 1.4] (see Section 3.3). In particular, we construct model structures on $E_{\infty}$ and strictly commutative algebras over motivic symmetric spectra.

At the end of the first subsection, we study model structures for algebras over motivic operads. This has not been considered so far. There is also one new (nonpositive!) model structure for $E_{\infty}$-operads in classical symmetric spectra, although the existence of such a model structure will not be too surprising to the expert. Namely, Proposition 3.9 also applies to the trivial site $\mathcal{C}$, that is simplicial-set valued symmetric $S^{1}$-spectra.

Later in this section, some further model structures and results related to simplicial presheaves and $H \mathbb{Z}$-modules are considered as well.

\subsection{Stable model structures}

Let $\mathcal{C}=(S m / S)_{\text {Nis }}$ and fix a cellular left proper model structure on $\Delta^{\mathrm{op}} \operatorname{PrShv}(\mathcal{C})$ which yields the Morel-Voevodsky [48] unstable homotopy category $H(S)$, and similarly for the pointed variant $\Delta^{\mathrm{op}} \operatorname{PrShv}(\mathcal{C})$. Throughout this section, we will work with the motivic injective model structure of [48] — or rather with its extension to simplicial presheaves as in [37] — which Hirschhorn ([29], see also [31, Corollary 1.6]) has shown to be cellular. We denote the generating cofibrations (resp. trivial cofibrations) by $I$ (resp. $J$ ). Besides being simplicial, cellular and proper, this model structure has two additional features which will be important in the sequel. First, the cofibrations are precisely the monomorphisms, in particular all objects are cofibrant. Second, it is a monoidal model category, that is it satisfies Hovey [33, Definition 4.2.6]. This follows 
because smashing with any object preserves weak equivalences, compare Morel and Voevodsky [48, Lemma 3.2.13], the author [31, Theorem 1.9] or Dundas, Röndigs and Østvær [17, Lemma 2.20]. Note that the second condition of [33] for being a monoidal model category is automatically satisfied because all objects are cofibrant.

We now fix an object $T$ of $\Delta^{\mathrm{op}} \operatorname{PrShv}(\mathcal{C})$. For many arguments below we may take an arbitrary $T$, but sometimes (eg in Theorems 3.6 and 3.15) we will need that $T \simeq S^{1} \wedge T^{\prime}$ for a suitable $T^{\prime}$, which holds in particular for $T=S^{1}$ and for $T=\mathbf{P}^{1}$. So we assume that $T \simeq S^{1} \wedge T^{\prime}$ for a suitable $T^{\prime}$ from now on, although many results do hold in greater generality.

We may apply [34, Theorem A.9, Definition 8.7] and Hirschhorn [30, Theorem 4.1.1] to get a stable model structure on the category of motivic symmetric spectra $\mathrm{Sp}^{\Sigma, T}(\mathcal{C})$ from the above unstable one on $\Delta^{\mathrm{op}} \operatorname{PrShv}(\mathcal{C})$. This model structure coincides with the one of [37, Theorem 4.15]. In particular, the motivic stable equivalences are those defined on [37, page 509], that is defined with respect to injective stably fibrant objects.

Theorem 3.1 (Hovey, Jardine) The above stable model structure on $\mathrm{Sp}^{\Sigma, T}(\mathcal{C})$ is simplicial, proper, cellular and monoidal.

Proof By [37, Theorem 4.15] we have a proper closed simplicial model structure. It remains to check the first condition of [33, Definition 4.2.6] for a monoidal model category, that is the pushout product axiom. For this we may apply [34, Theorem 8.11] as we have chosen a model structure on $\Delta^{\mathrm{op}} \operatorname{PrShv}(\mathcal{C})$ for which all objects are cofibrant, or directly quote [37, Proposition 4.19].

This stable model structure on spectra will be referred to as the projective stable model structure. The term "projective" refers to the way we obtained the stable structure from the unstable one, as the unstable model structure we started with really is an "injective"

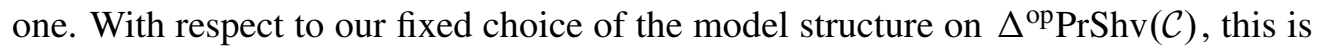
a motivic generalization of the model structure considered in [35, Theorem 3.4.4]. It will turn out that this model structure will not meet all our requirements, which is why we need to introduce a motivic version of the (positive) $S$-model $=$ flat model structure of [35] and Shipley [63]. The reasons for considering flat and positive model structures will become clear below. In the approach of Toën-Vezzosi, the reason for considering the flat model structure is that a motivic generalization of [63, Corollary 4.3] provides a tool to reduce [67, Assumption 1.1.0.4(2)] to [67, Assumption 1.1.0.3].

We will also need an injective stable model structure on motivic symmetric spectra, that is a model structure obtained by starting with the levelwise cofibrations and weak equivalences and then localize to obtain the stable model structure. This is necessary 
because some arguments below will use that the monomorphisms are cofibrations in a certain model structure, which means that for showing that a monomorphism $X \rightarrow Y$ is a weak equivalence it is sufficient to show that the quotient $Y / X$ is contractible, that is weakly equivalent to a point. This model structure has been first considered by Jardine [38].

Theorem 3.2 (Jardine) There is a model structure on $\operatorname{Sp}^{\Sigma, T}(\mathcal{C})$ with weak equivalences being the motivic stable equivalences and cofibrations being the levelwise monomorphisms. This model structure is simplicial and proper. It is called the injective stable model structure.

Proof See [38, Theorem 10.5] except for right proper, which follows from the right properness of the stable projective model structure which has more fibrations and the same weak equivalences.

Next, we establish a flat stable and a positive flat stable model structure. The identity morphisms between these four stable model structures, that is injective, flat, positive flat and projective, are all Quillen equivalences of simplicial model categories. Of course, it is also possible to establish projective and injective positive stable model structures, but we will not need these.

As in the classical case, there is a functor from symmetric sequences in $\Delta^{\mathrm{op}} \operatorname{PrShv}(\mathcal{C})$. to $\mathrm{Sp}^{\Sigma, T}(\mathcal{C})$ which is left adjoint to the forgetful functor. We denote it by $T \otimes-$, and it enjoys the same formal properties as the functor $S \otimes-$ in [35]. For the definition of $I-$ cof for a set of maps $I$ in a category, see eg [33, Definition 2.1.7].

Definition 3.3 A map is a motivic flat cofibration if it is in $T \otimes M-$ cof, where $M$ is the class of levelwise monomorphisms in symmetric sequences.

As we said above, we will also define "positive" variants of the model structures (at least for the flat one below), following [45, Definition 6.1, Definition 9.1 and page 484], which will be necessary to define a model structure on strictly commutative symmetric ring spectra further below. This variant has fewer cofibrations than the nonpositive (sometimes also called "absolute") model structure. In particular, the motivic symmetric sphere spectrum $\Sigma_{T}^{\infty} S^{0}$ is no longer cofibrant, so the usual contradiction related to the "Lewis paradoxon" does not appear (see eg [45, page 484]). Indeed, if one does not work with the positive model structure, then in the notation of Theorem 3.17 below the condition (2) of [30, Theorem 11.3.2] or equivalently [61, Lemma 2.3.(1)] that $U$ takes relative $L J$-cell complexes to stable weak equivalences will fail. Looking at the proofs for this condition (see in particular [63, Proposition 3.3] and [45, Lemma 15.5]) 
one sees how the positive model structure arises. The key point is that the argument in the proof of [45, Lemma 15.5] starting with "Since $\Sigma_{i}$ acts on $O(q)$ as a subgroup of $O(n i)$ " (read "on $\Sigma_{q}$ as a subgroup of $\Sigma_{n i}$ ") does not work if $n=0$.

Theorem 3.4 The category $\mathrm{Sp}^{\Sigma, T}(\mathcal{C})$ admits a model structure with the weak equivalences being the stable motivic equivalences and cofibrations being the motivic flat cofibrations. This model structure is simplicial, monoidal and proper, and we call it the flat stable model structure. There is also a positive flat stable model structure having the same stable weak equivalences and which enjoys the same properties (including the pushout product axiom), except that the motivic sphere spectrum is not flat positive cofibrant.

Proof To establish the flat and the flat positive model structure, there are various possible proofs. We proceed roughly by generalizing the corresponding results of [63]; see however the remark in parenthesis at the end of the proof. When adapting Shipley's definition of $I^{\prime}$ and $J^{\prime}$ to the motivic case, one must work with our above sets $I$ and $J$, of course. An alternative reference for [63, Proposition 1.2] which generalizes to the motivic case is Dwyer and Kan [18]; see also Rezk [54, Proposition 3.1.9]. The proof of [63, Proposition 1.3] goes through in the motivic case as well. Note that the model category on equivariant simplicial presheaves one obtains is left proper. Alternatively, one may deduce the motivic version of [63, Proposition 1.3] from [63, Proposition 1.2] using Hirschorn's [30] or Smith's [5] generalization of Bousfield localization.

[63, Proposition 2.1] is just the product model structure. When establishing the motivic generalizations of [63, Proposition 2.2 and Lemma 2.3], the arguments go through and yield a cofibrantly generated level model structure, which again is even cellular. To see this, observe that (both classical and) motivic symmetric spectra are cellular because simplicial presheaves are cellular, and so are products of cellular model categories. To check the three conditions of [30, Definition 12.1.1] for (motivic) symmetric spectra, first observe that the third condition is [34, Proposition A.4]. We then use the adjunction ( $T \otimes-, U=$ Forget) between symmetric sequences and symmetric spectra, where $U$ commutes with colims. The proof of the second condition is then similar to [34, Lemma A.2]. Finally, for establishing the first property one proceeds as in the proof of [34, Proposition A.8]. The argument there in fact slightly simplifies as we only have to consider one functor $T \otimes-$ rather than $F_{n}$ for fixed $n$ with intermediate considerations concerning $F_{m}$ for other values of $m$. The level flat model structure on motivic symmetric spectra is left proper because the injective stable model structure which has more cofibrations and the same weak equivalences is left proper. To obtain the motivic version of [63, Theorem 2.4], that is passing from the level to the stable model structure, one may apply Hirschhorn localization as done in [34, Definition 8.7] rather 
than checking the details corresponding to the ones in the proof of [63, Theorem 2.4], as we have shown that the flat level model structure is cellular and left proper. Hence the flat stable model structure is also left proper. Note that it is right proper because the stable projective model structure is right proper.

The proof that the positive model structure also exists again goes through in the motivic case. In more detail, the proof for the positive model structure is exactly the same, the only modification being that the motivic model structure generalizing the one found in [63, Proposition 2.1] is defined as taking on $\Sigma_{0}$-spaces the cofibrantly generated model structure with fibrations and weak equivalences being all morphisms. Then take the product model structure on motivic $\Sigma_{n}$-spaces for all $n \geq 0$ as before and proceed as in the nonpositive case. As the positive model structure has fewer cofibrations, it is also left proper. To show that the positive structure is right proper, note that the stronger statement of [35, Lemma 5.5.3 (2)] generalizes to the motivic case. To see this, one uses that the final argument of [35] carries over as the $\mathbf{A}^{1}$-local model structure on $\Delta^{\mathrm{op}} \operatorname{PrShv}(\mathcal{C})$ is right proper by [37, Theorem A.5], and that the proofs of [35, Theorem 3.1.14 and Lemma 3.4.15] do carry over.

To check that these model structures are monoidal, we must check the two conditions of [33, Definition 4.2.6]. The second condition in the nonpositive case is easy as the sphere spectrum is stably cofibrant because $* \rightarrow \operatorname{Spec}(S)_{+}$is cofibrant in $\Delta^{\mathrm{op} P r S h v}(\mathcal{C}) \bullet$ and $T \otimes-$ is left Quillen. Hence it remains to check the first condition, that is the pushout product axiom. The proof of [35, Theorem 5.3.7] goes through, and may even be simplified a bit, see Lemma 3.5 below, which also applies to the positive variant.

(It is possible to use the powerful machinery of Bousfield localization more systematically to obtain a different proof. For example, one may quote Heller [28, Theorem II.4.5] to obtain the global model structure for $\Sigma_{n}$-simplicial presheaves which correspond to [63, Proposition 1.3] and then impose cardinality bounds to see that this model structure is cofibrantly generated and even cellular. This is also done in [29, Theorem 4.9] who attributes this result to Smith, and in [5, Theorem 2.16]. Hirschhorn or Smith localization then yields the $\mathbf{A}^{1}$-local model structure on $\Sigma_{n}$-simplicial presheaves, with generating sets of (trivial) cofibrations different from the ones the approach of [63] yields. To see that the flat level model structure on $\operatorname{Sp}^{\Sigma, T}(\mathcal{C})$ is cellular, one may use the theorem of Jeff Smith on the existence of left Bousfield localizations for combinatorial model categories, which has been written up recently by Barwick [5, Theorem 4.7]. Still another variant would be to apply a more recent localization theorem of Bousfield as done in the appendix of [60], and this is certainly not the end of the list of variants of proofs.) 
Lemma 3.5 Both the flat stable and the positive flat stable model structure on $\mathrm{Sp}^{\Sigma, T}(\mathcal{C})$ satisfy the pushout product axiom.

Proof We only do the nonpositive case. In the positive case, the condition on cofibrations follows similarly, and the one for stable equivalences then follows from the corresponding property for the nonpositive model structure.

We start by observing that for any finite group $G$, the above model structure on $G$-objects in $\Delta^{\mathrm{op}} \operatorname{PrShv}(\mathcal{C})$. is monoidal because $\Delta^{\mathrm{op}} \operatorname{PrShv}(\mathcal{C})$. is monoidal and we have defined cofibrations and weak equivalences using the forgetful functor. It follows that the category of symmetric sequences in $\Delta^{\mathrm{op}} \operatorname{PrShv}(\mathcal{C}) \bullet$ is monoidal (see eg Harper [27, Theorem 12.2]). The stable flat model structure on $\operatorname{Sp}^{\Sigma, T}(\mathcal{C})$ has the same cofibrations as the level structure, so it remains to show that if given two cofibrations $f: A \rightarrow B$ and $g: X \rightarrow Y$ then if $f$ (or $g$ ) is a stable equivalence then so is $f \wedge g: A \wedge Y \amalg_{A \wedge X} B \wedge X \rightarrow B \wedge Y$. This can be shown exactly as in [35, Theorem 5.3.7(5)]. The argument goes through replacing as usual simplicial sets by simplicial presheaves and $S^{1}$ by $T$. In particular [35, Lemma 3.1.6] remains valid in this situation.

Note that similar to [35], the proof of this lemma provides a variant of the above proof that the stable projective model structure on $\operatorname{Sp}^{\Sigma, T}(\mathcal{C})$ satisfies the pushout product axiom.

Next, we wish to study operads $\mathcal{O}$ over motivic symmetric spectra. There are two approaches we are interested in: Simplicial operads, that is operads in simplicial sets for simplicial monoidal model categories, and internal operads in monoidal model categories. We sometimes apply one and sometimes the other point of view. Every operad in simplicial sets yields an internal operad in $\operatorname{Sp}^{\Sigma, T}(\mathcal{C})$ via the monoidal functor $\Sigma_{T}^{\infty}$, and a similar argument applies to the unstable case of simplicial presheaves. The converse is not true, but all operads we are interested in are simplicial ones. We will establish a theorem on the existence of model structures for arbitrary internal operads and stable positive model structures (see Theorem 3.6), and weaker results in the nonpositive case (see Proposition 3.9). The latter will be used when considering adjunctions of type $\left(\Sigma_{T}^{\infty}, E v_{0}\right)$ for $E_{\infty}$-objects.

In general, one of the standard ways to construct a model structure on a category $\mathcal{D}$ is to lift a cofibrantly generated model structure on a category $\mathcal{C}$ along a right adjoint in a free/forgetful-style adjunction $\mathcal{C} \rightleftarrows \mathcal{D}$, defining fibrations and weak equivalences in $\mathcal{D}$ by applying the forgetful functor. If this does yield a model structure, then the adjunction is Quillen and we say that $\mathcal{C}$ creates a model structure on $\mathcal{D}$. The main problem when checking the model axioms for $\mathcal{D}$ is that in one of the factorizations 
obtained by the small object argument it is not clear that certain relative cell complexes are weak equivalences. See eg [61, Lemma 2.3], [30, Theorem 11.3.2], Berger and Moerdijk $[8,2.5]$ and certain proofs below. One strategy for proving this is to establish a fibrant replacement functor; see eg the discussion in the remark after Proposition 3.11 below. Another strategy is to check the required property "by hand". If one is unable to successfully apply one of these two strategies, one may — as first done by Hovey [32] try to proceed by weakening the axioms of a model category in a suitable way, which leads to the notion of a semimodel category. See for example [32], Spitzweck [65] and Fresse [21, 12.1]; we will not pursue this approach.

The following theorem is a generalization of a result of Harper [26]. Compare also the article of Elmendorff and Mandell [20] which establishes a similar result for simplicial operads.

Theorem 3.6 Let $\mathcal{O}$ be any operad in $\operatorname{Sp}^{\Sigma, T}(\mathcal{C})$, and consider $\operatorname{Sp}^{\Sigma, T}(\mathcal{C})$ with the positive flat stable model structure. Assume that $T \simeq S^{1} \wedge T^{\prime}$ for some pointed object $T^{\prime}$. Then the forgetful functor from $\mathcal{O}$-alg to $\operatorname{Sp}^{\Sigma, T}(\mathcal{C})$ creates a model structure on $\mathcal{O}$-alg in the sense of [61, Lemma 2.3].

Proof The proof is along the lines of [26, Theorem 1.1]. We will indicate the nontrivial modifications to be made in the motivic setting. One proceeds by showing that the first condition of [61, Lemma 2.3] is satisfied. Using that transfinite compositions of acyclic monomorphisms are acyclic monomorphisms because they are the acyclic cofibrations for the model structure of Theorem 3.2, this boils down to show the motivic analog of [26, Proposition 4.4] about a certain morphism $j$. (Note that in the end we only care about symmetric sequences of symmetric spectra which are concentrated in degree zero, as discussed in [26, Section 7]) To prove the latter, using a filtration argument it is sufficient (notation taken from [26]) to show that $j_{t}$ is a weak equivalence for all $t$. For this we proceed as in [26, Proposition 4.29], using that there is a stable model structure on motivic spectra in which all levelwise monomorphisms are cofibrations, which exists thanks to Theorem 3.2. Hence we need motivic versions of [26, Propositions 4.28 and 4.29]. The proof of [26, Propositions 4.29] uses a five lemma argument which requires that smashing with $S^{1}$ detects stable weak equivalences, which is fine as $T \simeq T^{\prime} \wedge S^{1}$. Everything else now carries over to the motivic case. (Note that the positive model structure is used in [26, proof of Proposition 4.28], and looking at [26, Calculation 6.15] one sees exactly what fails for $m=0$.)

Remark The proof of Theorem 3.6 applies to other categories of symmetric spectra, that is starting with other model structures on $\Delta^{\mathrm{op}} \operatorname{PrShv}(\mathcal{C})$ than the motivic one. More precisely, let us consider the category $\Delta^{\mathrm{op}} \operatorname{PrShv}(\mathcal{M})$ of simplicial presheaves 
on an arbitrary small category $\mathcal{M}$. This category may be equipped with Heller's global injective model structure, which Hirschhorn has shown to be cellular and left proper [31, Theorem 1.4]. Thus we may consider the left (Hirschhorn-)Bousfield localization $L_{S} \Delta^{\mathrm{op}} \operatorname{PrShv}(\mathcal{M})$ with respect to an arbitrary set of morphisms $S$ in $\mathcal{M}$. The proof (see Theorem 3.12 below) that this model structure lifts to a model structure on algebras over an operad in $\Delta^{\mathrm{op}} \operatorname{PrShv}(\mathcal{M})$ goes through in this general setting provided we can show that the fibrant replacement functor in $\Delta^{\mathrm{op}} \operatorname{PrShv}(\mathcal{M})$ commutes with cartesian products. Now to generalize Theorem 3.6 to symmetric $T$-spectra build from $\Delta^{\mathrm{op}} \operatorname{PrShv}(\mathcal{M})$, we need to check that there is an induced stable injective model structure on symmetric spectra similar to the one of Jardine (see Theorem 3.2), that is one with all levelwise monomorphisms being cofibrations. This together with the fact that inverting $T \wedge-$ inverts $S^{1} \wedge-$ is all we really need to make the other proofs in this section work, in particular Theorem 3.6 and also Theorem 3.10 below, applying the techniques of $[35 ; 30 ; 26]$ in precisely the same way as in the case of motivic local model structures above.

Observe that the above forgetful functor admits the "free algebra" functor as a (Quillen) left adjoint, and also that both categories admit internal mapping spaces compatible under this adjunction. This will be generalized in Theorem 3.10 below.

We now shift our attention to nonpositive model structures. The next conjecture is inspired by results of Harper and Schwede. It applies in particular to simplicial $E_{\infty}$-operads.

Conjecture 3.7 Let $\mathcal{O}$ be any simplicial operad such that the action of $\Sigma_{n}$ on $\mathcal{O}(n)$ is free (by which we always mean objectwise and levelwise free away from the basepoint), and consider $\mathrm{Sp}^{\Sigma, T}(\mathcal{C})$ with the absolute flat or projective model structure. Then the forgetful functor from $\mathcal{O}-\operatorname{alg}\left(\operatorname{Sp}^{\Sigma, T}(\mathcal{C})\right)$ to $\operatorname{Sp}^{\Sigma, T}(\mathcal{C})$ creates a model structure on $\mathcal{O}-\operatorname{alg}\left(\operatorname{Sp}^{\Sigma, T}(\mathcal{C})\right)$ in the sense of [61, Lemma 2.3].

There is the following strategy of proof, which is an attempt of a motivic generalization of a variant of a proof for classical symmetric spectra as sketched in [60, Section III.4], notation are again as in [26]. In principle, it might be applicable to internal operads as well and not only to those with values in simplicial sets. As before, we are reduced to consider the pushout square of [26, Proposition 4.4]. It is shown in [27, Proposition 7.19] that the $\Sigma_{t}$-equivariant map $Q_{t-1}^{t} \rightarrow Y^{\wedge t}$ is an acyclic cofibration of symmetric spectra if $X \rightarrow Y$ is an acyclic cofibration. Using again the motivic generalization of [35, Theorem 5.3.7], this implies that the map $\mathcal{O}_{A}[t] \wedge Q_{t-1}^{t} \rightarrow \mathcal{O}_{A}[t] \wedge Y^{\wedge t}$ is a monomorphism and a weak equivalence for any $\mathcal{O}$-algebra $A$. Next, we show that the action of $\Sigma_{t}$ on the motivic symmetric spectra $\mathcal{O}_{A}[t]$ is free. By definition, the 
action of $\Sigma_{t}$ on $\mathcal{O}_{t}(A):=\bigvee_{k \geq 0} \mathcal{O}(k+t) \wedge \Sigma_{k} A^{\wedge k}$ as defined after [60, Remark 4.3] is free. Also by definition, $\mathcal{O}_{A}[t]$ is an explicit coequalizer of $\mathcal{O}_{t}(A)$ with respect to two $\Sigma_{t}$-equivariant maps coming from another spectrum with free $\Sigma_{t}$-action. Now one has to check that the action of $\Sigma_{t}$ on this quotient is also free. There are easy examples showing that this will not be true for arbitrary operads, so here one has to use the assumptions on the operad. Fresse even provided me with an example where the $\Sigma_{n}$-action on $\mathcal{O}(n)$ is free, but the action on $\mathcal{O}_{n}(A)$ is not. This is the gap one has to fill when using this strategy of proof. Hence the diagonal action of $\Sigma_{t}$ on $\mathcal{O}_{A}[t] \wedge Y^{\wedge t}$ is also free, and so are the ones on $\mathcal{O}_{A}[t] \wedge Q_{t-1}^{t}$ and on $\mathcal{O}_{A}[t] \wedge\left(Y^{\wedge t} / Q_{t-1}^{t}\right)$. That is, we have a cofiber sequence of $\Sigma_{t}$-free spaces

$$
\mathcal{O}_{A}[t] \wedge Q_{t-1}^{t} \rightarrow \mathcal{O}_{A}[t] \wedge Y^{\wedge t} \rightarrow \mathcal{O}_{A}[t] \wedge\left(Y^{\wedge t} / Q_{t-1}^{t}\right)
$$

for the injective model structure, which remains true after dividing out the free $\Sigma_{t}-$ action on all three objects. Indeed, taking coinvariants commutes with taking the cofiber as colims commute among each other, and as we have a model structure in which the monomorphisms are the cofibration, the cofiber of $\mathcal{O}_{A}[t] \wedge Q_{t-1}^{t} \rightarrow \mathcal{O}_{A}[t] \wedge Y^{\wedge t}$ is also the homotopy cofiber. The fact that $\mathcal{O}_{A}[t] \wedge\left(Y^{\wedge t} / Q_{t-1}^{t}\right)$ is weakly equivalent to a point remains true after dividing out the action of $\Sigma_{t}$ as the argument of [60, Proposition III.4.12] applies to general simplicial monoidal model categories, including motivic symmetric spectra. Besides the gap above, this seems to be the only place where it might be an advantage to restrict to operads defined in simplicial sets rather than in motivic symmetric $T$-spectra). Hence $\mathcal{O}_{A}[t] \wedge_{\Sigma_{t}} Q_{t-1}^{t} \rightarrow \mathcal{O}_{A}[t] \wedge \Sigma_{t} Y^{\wedge t}$ is an acyclic cofibration for the stable injective model structure, and now the proof can be finished as the one of Theorem 3.6.

Note that the recent preprint by Gorchinskiy and Guletski [25] contains a detailed discussion of techniques related to the problems above.

We can prove the above Conjecture 3.7 at least for the Barratt-Eccles operad, which will be sufficient for our purposes.

Definition 3.8 Let $\mathcal{W}$ be the Barratt-Eccles operad with values in the monoidal model category $\left(\Delta^{\text {op }}\right.$ Sets, $\left.\times, p t\right)$; see eg Berger and Fresse $[7,1.1 .5]$ and of course Barratt and Eccles [4]. It extends to an internal operad in $\operatorname{Sp}^{\Sigma, T}(\mathcal{C})$ via the functor $(-)_{+}$to pointed simplicial sets, the constant functor to $\Delta^{\mathrm{op}} \operatorname{PrShv}(\mathcal{C}) \bullet$ and $\Sigma_{T}^{\infty}$ to $\operatorname{Sp}^{\Sigma, T}(\mathcal{C})$ as all of these functors are monoidal. If we denote the internal operad in $\mathrm{Sp}^{\Sigma, T}(\mathcal{C})$ by $\mathcal{W}$ as well, the two notions of a $\mathcal{W}$-algebra in $\operatorname{Sp}^{\Sigma, T}(\mathcal{C})$ thus obtained coincide by definition and the above adjunctions. 
Observe that in simplicial degree zero, $\mathcal{W}$ is just the associative operad Ass, and a product of those in higher simplicial degrees.

We now prove the above conjecture for the operad $\mathcal{W}$. We do not know of a reference for this result even for classical symmetric spectra. The following proof also applies to other simplicial operads for which a decomposition pattern similar to the one below for $\mathcal{W}$ may be established.

Proposition 3.9 Conjecture 3.7 is true for $\mathcal{O}=\mathcal{W}$.

Proof According to the above strategy of proof, we must show that the coequalizer $\mathcal{W}_{A}[n]$ has a free action of $\Sigma_{n}$ for all $n \geq 0$. By definition [60, Construction III.4.8], [26, Proposition 4.6], the motivic symmetric spectrum with $\Sigma_{n}$-action $\mathcal{W}_{A}[n]$ is the coequalizer of

$$
\left(m, \mathcal{W}_{n}(\alpha)\right): \mathcal{W}_{n}(\mathcal{W}(A)) \rightrightarrows \mathcal{W}_{n}(A)
$$

where we have that $\mathcal{W}_{n}(A):=\bigsqcup_{p \geq 0} \mathcal{W}(n+p) \times_{\Sigma_{p}} A^{p}$ and $\mathcal{W}(A):=\mathcal{W}_{0}(A)$. The map $\alpha: \mathcal{W}(A)=\bigsqcup_{p \geq 0} \mathcal{W}(p) \times \Sigma_{p} A^{p} \rightarrow A$ is given by the $\mathcal{W}$-algebra structure of $A$. The map $m$ is given by the operad structure of $\mathcal{W}$ and will be described below, following [60, Section III.4]. By definition, the freeness of the $\Sigma_{n}$-action for a symmetric spectrum has to be checked objectwise (if the site is nontrivial, that is in the motivic case), and then for every symmetric spectrum levelwise, and in each level degreewise for the simplicial set. Also note that colimits in motivic symmetric spectra are constructed the same way (objectwise, levelwise, simplicially degreewise), and so are products in simplicial sets and more generally in simplicial presheaves, and similarly for the smash product in the pointed case. Finally, the smash product of a pointed simplicial set with a symmetric spectrum is given levelwise by the smash product of the pointed simplicial sets. All of this together implies that the whole argument really reduces to one of (pointed and even unpointed) simplicial sets, so we simplify our notation accordingly.

We now fix $n \geq 0$, and choose orbit decompositions of the sets $\Sigma_{n+k}=\mathcal{W}(n+k)_{0}$ with $\Sigma_{n}$-action by left multiplication for all $m \geq 0$. These decompositions yield decompositions of the simplicial sets $\mathcal{W}(n+k)$ for all $k \geq 0$, as $W(n+k)$ in simplicial degree $r$ is simply the $r+1$-fold product of $\Sigma_{n+k}$ with diagonal $\Sigma_{n}-$ action, and all simplicial structure maps are $\Sigma_{n+k}$-equivariant. So we only spell out the decompositions in simplicial degree zero. 
Recall that as a $\Sigma_{n}$-set with action given by left multiplication the set $\Sigma_{n+1}$ decomposes as a coproduct of $n+1$ copies of $\Sigma_{n}$, and inductively $\Sigma_{n+k}$ decomposes as a coproduct of $(n+k) \cdots(n+1)$ copies of $\Sigma_{n}$ for all $k \geq 0$. We now fix particular choices for these decompositions of all $\Sigma_{n+k}$ for a fixed given $n$ and all $k \geq 0$ once and for all, and consequently fix decompositions for all $\mathcal{W}(n+k)$.

In every simplicial degree $\mathcal{W}(n+k)$ is a finite set. The decompositions we choose may be written as products $\Sigma_{n+k}=\Sigma_{n} \times M_{k, n}$ where $\Sigma_{n}$ acts by left multiplication on the left factor and trivially on the right factor. Our decomposition is then determined by the following. For any positive integer $r$, any element $\sigma \in \Sigma_{r}$ is uniquely determined by $\sigma(1, \ldots, r)$. For $r=n+k$, we write $\sigma=\tau \times \rho$ with $\tau \in \Sigma_{n}$ being the element obtained by deleting all entries in $\sigma(1, \ldots, r)$ which are larger than $n$, and $\rho \in M_{k, n}$ being determined by where we insert these remaining elements $n+1, n+2, \ldots, n+k$ between the given permutation of $\tau(1, \ldots, n)$.

It is obvious that $\mathcal{W}_{n}(\alpha)$ maps copies of $\Sigma_{n}$ with respect to the above decomposition identically (that is not permuting the elements inside each copy of the $\Sigma_{n}$-set $\Sigma_{n}$ ) to copies of $\Sigma_{n}$. We will show that the same is true for the map $m$. Consequently, the coequalizer $\mathcal{W}_{A}[n]$ consists of free $\Sigma_{n}$-orbits as well, which finishes the proof.

According to [60], $m: \coprod_{s \geq 0} \mathcal{W}(n+s) \times \Sigma_{s}(\mathcal{W}(A))^{\times s} \rightarrow \bigsqcup_{r \geq 0} \mathcal{W}(n+r) \times \Sigma_{r} A^{r}$ is defined on the summand for a fixed $s \geq 0$ by the following composition of $\Sigma_{n}-$ equivariant maps:

$$
\begin{aligned}
& \mathcal{W}(n+s) \times \Sigma_{s} \mathcal{W}(A)^{\times s} \\
& \cong \coprod \mathcal{W}(n+s) \times \Sigma_{s}\left(\mathcal{W}\left(i_{1}\right) \times \cdots \times \mathcal{W}\left(i_{s}\right) \times \Sigma_{i_{1}} \times \cdots \times \Sigma_{i_{S}} A^{\times i_{1}+\cdots+i_{s}}\right) \\
& \stackrel{\cong}{\longrightarrow} \mathcal{W}(n+s) \times \Sigma_{s} \mathcal{W}(1) \times \cdots \times \mathcal{W}(1) \times \mathcal{W}\left(i_{1}\right) \times \cdots \times \mathcal{W}\left(i_{s}\right) \times \Sigma_{i_{1}} \times \cdots \times \Sigma_{i_{s}} A^{\times i_{1}+\cdots+i_{s}} \\
& \left.\rightarrow \coprod \mathcal{W}\left(n+i_{1}+\cdots+i_{S}\right) \times \Sigma_{i_{1} \times \cdots \times \Sigma_{i_{s}}} A^{\times i_{1}+\cdots+i_{s}}\right) \\
& \rightarrow \coprod \mathcal{W}(n+r) \times_{\Sigma_{r}} A^{\times r},
\end{aligned}
$$

where the last map is given by reindexing and the universal property of coproducts, and the second last map is given by the structure maps of the Barratt-Eccles operad $\mathcal{W}$. Now all four morphisms map free $\Sigma_{n}$-orbits identically to free $\Sigma_{n}$-orbits with respect to the $\Sigma_{n}$-decompositions introduced above. For the first, second and last morphism this is obvious. For the third map this can be checked using the equivariance condition of the operadic structure maps. To see this, again one first looks at the simplicial degree zero for which $\mathcal{W}(n+s)_{0}=\Sigma_{n+s}$, that is the associative operad Ass. Then generalize to higher degrees as explained above, which involves cartesian products of Ass, and argue componentwise. 
Once these results are established, one may deduce the motivic variant of [26, Theorem 1.4]. Namely, we have the following.

Theorem 3.10 Let $f: \mathcal{O} \rightarrow \mathcal{O}^{\prime}$ be a morphism of operads and consider the lifts of the flat positive stable model structure on $\operatorname{Sp}^{\Sigma, T}(\mathcal{C})$ to $\mathcal{O}-\operatorname{alg}\left(\operatorname{Sp}^{\Sigma, T}(\mathcal{C})\right)$ and $\mathcal{O}^{\prime}-\operatorname{alg}\left(\operatorname{Sp}^{\Sigma, T}(\mathcal{C})\right)$. Then $f$ induces an Quillen adjunction (enriched over $\Delta^{\mathrm{op}}$ Sets)

$$
f_{*}: \mathcal{O}-\operatorname{alg}\left(\operatorname{Sp}^{\Sigma, T}(\mathcal{C})\right) \rightleftarrows \mathcal{O}^{\prime}-\operatorname{alg}\left(\operatorname{Sp}^{\Sigma, T}(\mathcal{C})\right): f^{*}
$$

which is a Quillen equivalence if $f$ is a stable weak equivalence in every operadic degree.

Proof By construction of our motivic model structures, the proof of [26, Theorem 1.4] carries over. Enrichments are not mentioned in [26], but the arguments given there immediately show they behave as well as expected.

In particular, the model categories of $E_{\infty}$-algebras and strictly commutative monoids in $\operatorname{Sp}^{\Sigma, T}(\mathcal{C})$ are Quillen equivalent.

We continue to study the absolute (nonpositive) situation. If one can not prove Conjecture 3.7 for a given operad $\mathcal{O}$ using the strategy discussed above, a different approach might be to first look at the level model structure as in the following result.

Proposition 3.11 There is a projective level model structure on $\operatorname{Sp}^{\Sigma, T}(\mathcal{C})$ with fibrations and weak equivalences defined levelwise. This model structure lifts to $\mathcal{O}_{-}$ $\operatorname{alg}\left(\operatorname{Sp}^{\Sigma, T}(\mathcal{C})\right)$ for any operad $\mathcal{O}$.

Proof In the classical case (ie for the trivial site), the projective level model structure on $\mathrm{Sp}^{\Sigma, T}(\mathcal{C})$ is introduced in [35] and the positive variant in [60;63;45]. These level model structures are cofibrantly generated with respect to acyclic cofibrations $I$ and $J$ (resp. $I^{+}$and $J^{+}$). To show that it lifts to $\mathcal{O}-\operatorname{alg}\left(\operatorname{Sp}^{\Sigma, T}(\mathcal{C})\right.$ ), as before the only thing that one has to check is that any map in $\mathcal{O} J$-cell is a weak equivalence. For this one may proceed similarly to [19, Lemma VII.5.6]. Namely, the geometric realizations of the maps in $\mathrm{J}$ are ( $\Sigma^{\infty}$ of) inclusions of deformation retracts. Furthermore, these are stable under the free functor $\mathcal{O}$, under pushouts in $\mathcal{O}-\operatorname{alg}\left(\operatorname{Sp}^{\Sigma, T}(\mathcal{C})\right.$ ) (by refining an argument of [33, Proposition 2.4.9], as Mandell kindly explained to me) and under sequential colimits. Note that geometric realization does preserve colimits, and a map in $\operatorname{Sp}^{\Sigma, T}(\mathcal{C})$ is a level equivalence if and only if its geometric realization is.

In the motivic case (that is for the nontrivial site), the argument has to be refined a bit. Looking at diagram categories, one obtains global level (absolute and positive) 
model structures on $\mathrm{Sp}^{\Sigma, T}(\mathcal{C})$ with $J$ consisting of inclusions of deformation retracts (objectwise, in the classical sense) and consequently on $\mathcal{O}-\operatorname{alg}\left(\operatorname{Sp}^{\Sigma, T}(\mathcal{C})\right.$ ). To obtain the $\mathbf{A}^{1}$-local level model structures, one applies Bousfield-Hirschhorn localization to $\operatorname{Sp}^{\Sigma, T}(\mathcal{C})$ and to $\mathcal{O}-\operatorname{alg}\left(\operatorname{Sp}^{\Sigma, T}(\mathcal{C})\right)$ to a suitable set $S$, which yields exactly the cofibrantly generated motivic level model structure of [34, Theorem 8.2] on $\mathrm{Sp}^{\Sigma, T}(\mathcal{C})$. For $\mathcal{O}-\operatorname{alg}\left(\operatorname{Sp}^{\Sigma, T}(\mathcal{C})\right)$ one applies Bousfield-Hirschhorn localization with respect to the set obtained by applying the free functor $\mathcal{O}$ to $S$.

Note that if we could apply Bousfield-Hirschhorn or Bousfield-Smith localization with respect to a suitable set of stable weak equivalences, this would yield an alternative proof of Conjecture 3.7. The problem both with Hirschhorn's and Smith's approach is that $\mathcal{O}-\operatorname{alg}\left(\operatorname{Sp}^{\Sigma, T}(\mathcal{C})\right)$ with respect to the level model structure is not left proper in general, as one sees looking eg at $\mathcal{O}=\mathrm{Comm}$. We will not pursue this approach in our article.

Remark There is a model structure on the category of operads in simplicial sets given by [8, Theorem 3.2 and Example 3.3.1], in particular weak equivalences and fibrations are defined on the underlying simplicial sets. Note that this model structure is different from the one of [54]. Looking at $[8,4.6 .4]$ for classical symmetric spectra, the argument in the proof of [8, Theorem 3.5(a)] applies to simplicial model categories in general, defining $\mathcal{E}_{f}$ in the category of simplicial sets and applying SM7. In particular, we may apply this theorem to a fibrant replacement map $f: X \rightarrow X_{\text {fib }}$ in $\operatorname{Sp}^{\Sigma, T}(\mathcal{C})$ and a cofibrant model $P$ for the $E_{\infty}$-operad with respect to the model structure of [8, Example 3.3.1]. Consequently, if we can show (which we can not) the hypothesis in [8, Theorem 3.5(a)] holds, namely that $f^{\wedge n}$ is a trivial cofibration for all $n>0$, then we have constructed a fibrant replacement for any object in $\mathcal{O}-\operatorname{alg}\left(\operatorname{Sp}^{\Sigma, T}(\mathcal{C})\right)$. Note however that this construction is not functorial. Hence it is not clear if we may apply a variant of an argument of Quillen [53], see eg [54, Proposition 3.1.5] or Schwede [59, B.2 and B.3], to lift the model structure of $\operatorname{Sp}^{\Sigma, T}(\mathcal{C})$ to a model structure on $\mathcal{O}-\operatorname{alg}\left(\operatorname{Sp}^{\Sigma, T}(\mathcal{C})\right)$. In general, in a monoidal model category in which all objects are cofibrant, the hypothesis holds as can be shown by an easy induction. More generally, to check the hypothesis in a monoidal model category (a similar argument then presumably applies to simplicial model categories and simplicial instead of internal operads), [8, Remark 3.6] claims it is enough to have a set of generating trivial cofibrations having cofibrant domains. Classical symmetric spectra with the (absolute $=$ nonpositive) flat or projective model structure satisfy this property and are monoidal model categories. But it is not clear why this helps as Harper gave an easy example of a cofibrantly generated monoidal model category whose generating acyclic cofibrations have cofibrant domains, but where the above property for $f^{\wedge n}$ fails already 
for $n=2$. In short, the techniques of [8] do not provide an absolute stable model structure for $\mathcal{O}$-algebras in symmetric spectra with $\mathcal{O}$ a simplicial or internal cofibrant operad.

\subsection{Unstable model structures}

We will now establish a variant of Theorem 3.6 for spaces rather than spectra, that is an "unstable model structure", as well as Quillen adjunctions between unstable and stable model categories. We also show that a motivic generalization of the axioms of Goerss-Hopkins holds. For the sake of completeness, we recall that there is also an unstable projective model structure on simplicial presheaves, starting with fibrations and weak equivalences defined objectwise and then localizing with respect to the Nisnevich topology and the affine line as in the unstable injective case above. The identity functor obviously induces Quillen equivalences between the global (and hence between the local) unstable projective and injective model structure. Note that the unstable local projective model structure is also simplicial, cellular and monoidal; see Blander [12] and [31]. We will only use the unstable injective model structure in the sequel.

Theorem 3.12 For any operad $\mathcal{O}$, the injective motivic model structures on simplicial presheaves lifts via the forgetful functor to the category $\mathcal{O}-\operatorname{alg}\left(\Delta^{\mathrm{op}} \operatorname{PrShv}(\mathcal{C}) \bullet\right)$ of $\mathcal{O}$-algebras in this model category.

Proof We have a motivic fibrant replacement functor in $\mathcal{O}-\operatorname{alg}\left(\Delta^{\mathrm{op}} \operatorname{PrShv}(\mathcal{C}) \bullet\right)$ as the usual fibrant replacement functor commutes with products, so the argument of Quillen discussed above applies; see also [54, Proposition 3.2.5]. The existence of the motivic (that is $\mathbf{A}^{1}$-local) fibrant replacement functor on $\mathcal{O}-\operatorname{alg}\left(\Delta^{\mathrm{op}} \operatorname{PrShv}(\mathcal{C}) \bullet\right.$ follows from the existence of a fibrant replacement functor on $\Delta^{\mathrm{op}} \operatorname{PrShv}(\mathcal{C})$. which commutes with $\times$. More precisely, we have that both motivic fibrant replacement functors constructed in [48, Lemma 2.3.20, Lemma 3.2.6] commute with finite limits by [48, Theorem 2.1.66, page 97]. (Note that for the special case $\mathcal{O}=\mathcal{W}$, we may of course alternatively adapt the above proof for $\mathrm{Sp}^{\Sigma, T}(\mathcal{C})$.)

Theorem 3.13 There is a Quillen adjunction

$$
\Sigma_{T}^{\infty}: \Delta^{\mathrm{op}} \operatorname{PrShv}(\mathcal{C}) \bullet \rightleftarrows \mathrm{Sp}^{\Sigma, T}(\mathcal{C}): E v_{0},
$$

where both functors are strong monoidal. Here $\Delta^{\mathrm{op}} \operatorname{PrShv}(\mathcal{C})$. is equipped with the above injective local model structure, and $\mathrm{Sp}^{\Sigma, T}(\mathcal{C})$ is equipped with the projective, flat or injective stable model structure built from it as discussed above. 
Proof The adjunction was already established in Section 1. By [34, Proposition 8.5], the functor $E v_{0}$ is right Quillen with respect to the projective level structure on $\mathrm{Sp}^{\Sigma, T}(\mathcal{C})$, and consequently with respect to all stable model structures mentioned in the Theorem.

The following result shows why we it was important to establish absolute rather than only positive model structure on $\mathcal{O}-\operatorname{alg}\left(\operatorname{Sp}^{\Sigma, T}(\mathcal{C})\right)$.

Corollary 3.14 Let $\mathcal{O}$ be an operad such that the forgetful functor to the category $\mathcal{O}-\operatorname{alg}\left(\Delta^{\mathrm{op}} \operatorname{PrShv}(\mathcal{C}) \bullet\right)$ creates an absolute stable projective resp. flat model structure on the latter, eg $\mathcal{O}=\mathcal{W}$. Then the Quillen adjunction of Theorem 3.13 induces a Quillen adjunction

$$
\Sigma_{T}^{\infty}: \mathcal{O}-\operatorname{alg}\left(\Delta^{\mathrm{op}} \operatorname{PrShv}(\mathcal{C}) \bullet\right) \rightleftarrows \mathcal{O}-\operatorname{alg}\left(\operatorname{Sp}^{\Sigma, T}(\mathcal{C})\right): E v_{0}
$$

for the stable projective resp. flat model structures on $\mathcal{O}-\operatorname{alg}\left(\Delta^{\mathrm{op}} \operatorname{PrShv}(\mathcal{C}) \bullet\right)$.

Proof This follows immediately from Theorem 3.13 and the definition of the model structures on $\mathcal{O}$-algebras via forgetful functors.

\subsection{The axioms of Goerss-Hopkins}

We now show that some of the above model structures feed into the motivic version of the obstruction theory machine for $E_{\infty}$-ring spectra of Goerss and Hopkins [23]. The machinery of Toën and Vezzosi [67] will be discussed further below. As we already said in the introduction, this result has been obtained independently by Østvær.

Theorem 3.15 Both the flat and the projective stable positive model structures on $\mathrm{Sp}^{\Sigma, T}(\mathcal{C})$ satisfy the motivic analog of the five axioms in [23, 1.1 and 1.4].

Proof Everything has been shown above already except that the generating cofibrations and the generating acyclic cofibrations can be chosen to have cofibrant source and condition (5). As every object in $\Delta^{\mathrm{op}} \operatorname{PrShv}(\mathcal{C})$. is cofibrant, the sources of the generating cofibrations and of the generating acyclic cofibrations for the stable flat model structure on $\operatorname{Sp}^{\Sigma, T}(\mathcal{C})$ are cofibrant because $T \otimes-$ is left Quillen, hence this holds in particular for the stable flat positive model structure. Consequently, the same is true for the model structure of operads as the proof of Theorem 3.6 shows that the forgetful functor $\mathcal{O}_{-}$alg $\rightarrow \mathrm{Sp}^{\Sigma, T}(\mathcal{C})$ is right Quillen. To show the claim for the generating acyclic cofibrations, note that essentially the same argument goes through for those, since the domains of the motivic variant of the class $K$ 
of [35, Definition 4.3.9] also have cofibrant sources because $E v_{n}$ is right Quillen for the flat level model structure, hence $F_{n}$ preserves cofibrations, and the model structures satisfy the pushout product axiom. Alternatively, we may quote [30, Proposition 4.5.1]. Condition (5) follows again from the motivic variants of Harper's results discussed above. Namely, one first uses [26, Proposition 4.28(a)] applied to the cofibration $*=\operatorname{Spec}(k) \rightarrow X$ to see that $X^{\wedge t}$ is then also cofibrant, and then applies the motivic variant of [26, Proposition 4.29(b)] for equivariant symmetric sequences concentrated in degree zero, that is equivariant symmetric spectra, equipped with the model structure of [26, Section 4] for which the weak equivalences are defined by forgetting the $\Sigma_{t}-$ action.

\subsection{HA-contexts}

We now establish model structures for commutative ring spectra and algebras over those and establish the properties required in the axioms of [67]. For this, we consider the category $\operatorname{Comm}\left(\mathrm{Sp}^{\Sigma, T}(\mathcal{C})\right)$ of commutative unital monoids in $\mathrm{Sp}^{\Sigma, T}(\mathcal{C})$. The notation Comm is taken from [67], further below we write AbMon instead which is more consistent with unstable notation. The forgetful functor $U: \operatorname{Comm}\left(\operatorname{Sp}^{\Sigma, T}(\mathcal{C})\right) \rightarrow \operatorname{Sp}^{\Sigma, T}(\mathcal{C})$ has a left adjoint $L$, namely the obvious motivic variant of [63, Section 3]. For $R \in \operatorname{Comm}\left(\operatorname{Sp}^{\Sigma, T}(\mathcal{C})\right)$, we define the monoidal category $R-\bmod$ in the usual way.

Theorem 3.16 Consider the stable flat absolute or positive model structure on $\mathrm{Sp}^{\Sigma, T}(\mathcal{C})$, and let $R$ be an arbitrary object in $\operatorname{Comm}\left(\operatorname{Sp}^{\Sigma, T}(\mathcal{C})\right)$. Then there is a model structure on $R-\bmod \left(\operatorname{Sp}^{\Sigma, T}(\mathcal{C})\right)$ where the weak equivalences and fibrations are defined using the forgetful functor $R-\bmod \left(\operatorname{Sp}^{\Sigma, T}(\mathcal{C})\right) \rightarrow \operatorname{Sp}^{\Sigma, T}(\mathcal{C})$ where the latter is equipped with the (absolute or positive) flat model structure. These model structures are monoidal, proper and combinatorial. Moreover, we have Quillen adjunctions

$$
\begin{gathered}
R \wedge-:\left(\operatorname{Sp}^{\Sigma, T}(\mathcal{C})\right) \rightleftarrows R-\bmod \left(\mathrm{Sp}^{\Sigma, T}(\mathcal{C})\right): U, \\
U: R-\bmod \left(\operatorname{Sp}^{\Sigma, T}(\mathcal{C})\right) \rightleftarrows \operatorname{Sp}^{\Sigma, T}(\mathcal{C}): \operatorname{Map}(R,-),
\end{gathered}
$$

with respect to the flat model structure, where we assume that $R$ is cofibrant for the second Quillen adjunction.

Proof The existence of the model structures follow from the model structures of Theorem 3.4 by applying either Kan's lifting theorem [30, Theorem 11.3.2] using $R \wedge-$ as left adjoint, or the essentially equivalent [61, Theorem 4.1(2)]. We do not know if the monoid axiom holds (compare [34, Page 107]), but it is sufficient to check the second condition of [30, Theorem 11.3.2] (or equivalently the first condition of [61, Lemma 2.3]), the first one is obvious. If $R$ is cofibrant, then as the stable flat model 
structure is monoidal, the claim follows as explained in [61, Remark 4.2]. In fact, the monoid axiom probably holds in our case by some variant of this argument, but we will not need this.

For arbitrary $R$ one must use the motivic generalization of [35, Theorem 5.3 .7 (5)], compare the classical proof of [60, Theorem IV.1.4]. Note that [67, Assumption 1.1.0.2] requires the model structure also for noncofibrant $R$. Both left and right properness in $R-\bmod$ follow from left and right properness of $\mathrm{Sp}^{\Sigma, T}(\mathcal{C})$. Right properness is immediate as the weak equivalences and fibrations in $R$-mod are defined by the forgetful functor the left proper model category $\mathrm{Sp}^{\Sigma, T}(\mathcal{C})$. To show left properness, one uses that the generating cofibrations are level monomorphisms, hence so are all relative cell complexes built from them. Now use [34, Corollary 2.1.15].

That the two adjunctions exists follows in the standard way. That the first adjunction is Quillen follows from the definition of the model structures involved. To prove that the second adjunction is Quillen, one must simply show that for cofibrant $R$ the functor $\operatorname{Map}(R,-)$ preserves fibrations and trivial fibrations, which immediately follows from the fact that $\mathrm{Sp}^{\Sigma, T}(\mathcal{C})$ is a monoidal model category.

It seems possible to prove the above result for the projective variant as well, but we will not need this. Compare also [17, Section 4] for similar results about motivic functors.

Remark One must show that the above model structure is combinatorial as desired by Toën and Vezzosi. For this, observe that simplicial sets are small [33, Lemma 3.1.1], hence so are diagram categories over it (see also [61, Remark 2.4]). For how to use this to show that symmetric spectra are also small and so are motivic symmetric spectra using a similar argument; see [35, Proposition 3.2.13]. This shows that the category of symmetric spectra is locally presentable, and as all model structures we consider are cofibrantly generated, they are therefore all combinatorial. (Note that this also shows that instead of choosing quite explicit sets of generating (trivial) cofibrations for the above model structures, one might instead take instead all cofibrations resp. trivial cofibrations with codomains bounded by $\alpha$ (a cardinal that in a suitable sense is large enough with respect to $\mathrm{Sp}^{\Sigma, T}(\mathcal{C})$ ) as the set of generating (trivial) cofibrations. Then it remains to check that the (trivial) fibrations are indeed those of the model structure, that is it is enough to check the lifting property of a (trivial) on these sets. In most examples this is not hard to see.)

For $R \in \operatorname{Comm}\left(\mathrm{Sp}^{\Sigma, T}(\mathcal{C})\right)$, we denote the category of commutative $R$-algebras by $R-\operatorname{Comm}\left(\operatorname{Sp}^{\Sigma, T}(\mathcal{C})\right)$. We have a forgetful functor $U: \operatorname{Comm}\left(\operatorname{Sp}^{\Sigma, T}(\mathcal{C})\right) \rightarrow \operatorname{Sp}^{\Sigma, T}(\mathcal{C})$. 
Theorem 3.17 The stable flat positive model structure on $\operatorname{Sp}^{\Sigma, T}(\mathcal{C})$ creates a proper combinatorial model structure on $\operatorname{Comm}\left(\operatorname{Sp}^{\Sigma, T}(\mathcal{C})\right)$ where $f$ is a weak equivalence (resp. fibration) if and only $U f$ is. If $R \in \operatorname{Comm}\left(\mathrm{Sp}^{\Sigma, T}(\mathcal{C})\right)$, then the same is true for $R-\operatorname{Comm}\left(\operatorname{Sp}^{\Sigma, T}(\mathcal{C})\right)$.

Proof The existence of the cofibrantly generated model structure on $\operatorname{Comm}\left(\mathrm{Sp}^{\Sigma, T}(\mathcal{C})\right)$ follows from Theorem 3.6 applied to the operad Comm.

(In particular, this provides an alternative to the proof of Shipley [63] which relies on [30, Theorem 11.3.2]; see also Schwede [60, Theorem A.1.4], Hovey, Shipley and Smith [35, Lemma 2.3] and Mandell, May, Schwede and Shipley [45, Proposition 5.13]. Note also that [60] assumes that $U$ commutes with filtered colims which implies that all small colims exist, which is an assumption in [30]. As all objects are small, the only nontrivial thing of the assumptions that is left for Shipley to check is that $L J$-cell complexes - recall that $J$ are the generating trivial cofibrations - are stable weak equivalences, which is not so easy and relies on her [63, Propositions 3.3 and 3.4].)

As discussed above, it is easy to see that the underlying category is locally presentable, and thus the model category is combinatorial.

By the same formal argument as the one in the proof of [63, Theorem 3.2], the model structure on $R-\operatorname{Comm}\left(\operatorname{Sp}^{\Sigma, T}(\mathcal{C})\right)$ follows from the one of $\operatorname{Comm}\left(\operatorname{Sp}^{\Sigma, T}(\mathcal{C})\right)$. It remains to show properness. The model structures on $\operatorname{Comm}\left(\operatorname{Sp}^{\Sigma, T}(\mathcal{C})\right)$ and more generally on $R-\operatorname{Comm}\left(\mathrm{Sp}^{\Sigma, T}(\mathcal{C})\right)$ are right proper by the argument of the proof of Proposition 4.7 in [63] as we can prove the motivic generalization of the variant of [35, Lemma 5.5.3(2)] for positive level fibrations. To show that the proof of right properness of [35, Lemma 5.5.3(2)] generalizes to the motivic case, one uses that the final argument carries over as the $\mathbf{A}^{1}$-local model structure on $\Delta^{\text {op }} \operatorname{PrShv}(\mathcal{C})$ is right proper [37, Theorem A.5], and that the proofs of [35, Theorem 3.1.14 and Lemma 3.4.15] do carry over. To check that the model structure is also left proper, one uses the motivic analogue of [35, Corollary 5.3.10] — which follows from the motivic generalization of [35, Theorem 5.3.7] and Ken Brown's Lemma - and then proceeds as in the proof of [63, Proposition 4.7].

Moreover, we have the following, which yields the Axioms 1.1.0.3 and 1.1.0.4(2) in the definition of a $H A$-context as considered by Toën and Vezzosi [67]. Observe that Axiom 1.1.0.3 is not a formal consequence of the property "monoidal" established in Theorem 3.16. 
Proposition 3.18 Consider the stable flat positive model structure on $\operatorname{Sp}^{\Sigma, T}(\mathcal{C})$, which we already have shown to be monoidal.

(i) The monoidal model structure on $\mathrm{Sp}^{\Sigma, T}(\mathcal{C})$ is symmetric monoidal.

(ii) Let $R \in \operatorname{Comm}\left(\operatorname{Sp}^{\Sigma, T}(\mathcal{C})\right)$. Then for any cofibrant object $M \in R-\bmod$, the functor $-\wedge_{R} M$ preserves weak equivalences. For any cofibrant object $B \in R-\operatorname{Comm}\left(\operatorname{Sp}^{\Sigma, T}(\mathcal{C})\right)$, the functor $B \wedge_{R}-: R-\bmod \rightarrow B$-mod preserves weak equivalences.

Proof Part (i) is clear. Part (ii) follows from a motivic generalization of the ideas of [63, Section 4] which can be carried out thanks to the results we already established. More precisely, observe that using the motivic generalization of [63, Corollary 4.3] (which holds since [63, Proposition 4.1] generalizes to the motivic situation), we have that Assumption 1.1.0.4(2) reduces to Assumption 1.1.0.3, which is a motivic generalization of [35, Lemma 5.4.4]. This motivic generalization holds as we have already observed that [35, Theorem 5.3.7, Corollary 5.3.10] generalize to the motivic case.

The above results establish [67, Assumptions 1.1.0.1-1.1.0.4] for motivic symmetric spectra $\operatorname{Sp}^{\Sigma, T}(\mathcal{C})$. The nonunital variant (take away the index 0 in the definition of the free functor $L$ ) mentioned in [67, 1.1.0.4.(1)] (compare also Remark 1.1.0.5 of loc. cit.) follows again from Theorem 3.6 applied to the reduced commutative operad $\mathrm{Comm}_{n u}$ with $\left(\mathrm{Comm}_{n u}\right)_{0}=\Sigma_{T}^{\infty}(p t)$ when considered as an internal operad in $\operatorname{Sp}^{\Sigma, T}(\mathcal{C})$. For classical symmetric spectra, this was already stated in [67, Example (4) following Remark 1.1.0.7].

Remark In [67, page 20, Example (4)], it is stated that Assumptions 1.1.0.1-1.1.0.4 hold for symmetric spectra (that is $\mathcal{C}$ being the trivial category in our setting) by the results of [63], although some of the relevant points are not explained in full detail. Most of this is carried out in the arguments above. It remains to show that all categories involved are locally presentable. The standard references for locally presentable categories are Adámek and Rosický [1] and Borceux [14]. I do not know a reference for a detailed proof why symmetric spectra are locally presentable, but this easily follows from the smallness property as explained above. The reason for this condition is that [67] quote unpublished work from Smith - the relevant parts are now available thanks to [5] — in order to ensure that certain localized model structures exist; see eg [67, Section 1.3.1]. Our localization arguments in the first half rely on the published work of [30] on cellular model categories instead, but the arguments of Smith do apply just as well. So it is rather a matter of personal taste if one works with Smith's or with Hirschhorn's version of Bousfield localization. 
Note that Assumption 1.1.0.4(2) (which is part of Proposition 3.18(ii)) is probably not true for the positive projective model structure but only for the positive flat model structure (compare also [45, Theorem 14.5]). Here is why [63, Proposition 4.1] (which is an ingredient of the proof of [63, Corollary 4.3]) fails for the projective model structure already for $R=S$. In the notation of [63], the proof uses that the maps of $\mathbf{P}_{S}\left(S^{+} I\right)$ are $S$-cofs, which is deduced from the fact that the maps in $\mathbf{P}\left(I^{l+}\right)$ are coproducts of monomorphisms of symmetric sequences. It is not clear that the corresponding maps $\mathbf{P}_{S}\left(S^{+} I\right)$ are stable cofibrations for the projective model structure.

\section{5 $H \mathbb{Z}$-modules}

Theorem 3.16 will be an ingredient in the proof of Theorems 1.1 and 1.2, but will only be used in the case where $T=S^{1}$ and $R=H \mathbb{Z}$ is the usual simplicial Eilenberg Mac Lane spectrum considered as objectwise constant simplicial presheaf. Such an object $R$ is called flat resp. projective if it is cofibrant in $\operatorname{Sp}^{\Sigma, T}(\mathcal{C})$ with respect to the flat resp. projective stable model structure. So if you do not care about [67], the following lemma allows you to take a shortcut.

Lemma 3.19 Both the classical and the objectwise constant motivic $S^{1}$-spectrum $H \mathbb{Z}$ are flat.

Proof It suffices to show that $H \mathbb{Z}$ is flat as a classical symmetric spectrum. Using the adjunction between constant presheaves and presheaves, it follows that the presheaf of $S^{1}$-spectra $H \mathbb{Z}$ is cofibrant for the global flat stable model structure as well, and further (motivic left) localizations do not change the cofibrations. But as a classical symmetric spectrum, $H \mathbb{Z}$ is flat cofibrant; see [60].

It remains to lift the model structures on "naive" $H \mathbb{Z}$-modules and $\mathrm{Ch}(\mathbf{A b})$ to motivic (=Nisnevich- $\mathbf{A}^{1}$-local) model structures on presheaves of those as well, using similar techniques as before. For this we first recall the relevant classical model structures.

Theorem 3.20 The category $\mathrm{Ch}(\mathbf{A b})$ has a model structure with weak equivalences being the quasi-isomorphisms and fibrations the epimorphisms. The full subcategory $\mathrm{Ch}(\mathbf{A b})_{\geq 0}$ has a model structure with weak equivalences being the quasi-isomorphisms and fibrations the epimorphisms in degree greater than or equal to 1 . The inclusion incl and the good truncation $\tau_{\geq 0}$ form a Quillen adjunction between these model categories. The category $\Delta^{\mathrm{op}} \mathbf{A b}$ has a model structure with weak equivalences and fibrations being the weak equivalences and fibrations of the underlying simplicial sets. The Dold-Kan correspondence between $\Delta^{\mathrm{op}} \mathbf{A b}$ and $\mathrm{Ch}(\mathbf{A b})_{\geq 0}$ is an isomorphism of model categories. All three model categories are cofibrantly generated and left proper. 
Proof For the cofibrantly generated model structures, see [53] for $\mathrm{Ch}(\mathbf{A b})_{\geq 0}$, see [33, Theorem 2.3.11] for $\mathrm{Ch}(\mathbf{A b})$ and [33, Theorem III.2.8 and Theorem III.2.12] for $\Delta^{\mathrm{op}} \mathbf{A b}$ (or use the lifting argument from [61, Lemma 2.3.(2)] as before for the latter, recalling that there is a fibrant replacement functor in $\Delta^{\mathrm{op}}$ Sets which preserves products). The claims about the Quillen adjunction resp. equivalence are now straightforward. Left properness for $\mathrm{Ch}(\mathbf{A b})$, and hence for the other two model categories as well, follows from [33, Proposition 3.2.9].

Theorem 3.21 Theorem 3.20 generalizes to the corresponding motivic model categories.

Proof Use the same techniques as before. First, pass to diagram categories, that is presheaves with values in the above model categories, and then Bousfield-HirschhornSmith localize with respect to the Nisnevich topology and to the affine line. Note that $\mathrm{Ch}(\mathbf{A b})$ is cellular by [33, Lemma 2.3.2].

We will need one more auxiliary model category, namely "naive" $H \mathbb{Z}$-modules, taken from another article of Schwede and Shipley [62, Definition B.1.1]. Again, we first explain the classical case. Once more using the above techniques, everything generalizes to the motivic case using [30, Proposition 12.1.5 and Theorem 13.1.14] and Bousfield-Hirschhorn localization. We omit the details.

Definition 3.22 A naive $H \mathbb{Z}$-module is a collection of pointed simplicial sets $\left\{M_{n}\right\}_{n \geq 0}$ and associative and unital action maps $(H \mathbb{Z})_{p} \wedge M_{q} \rightarrow M_{p+q}$. A morphism of naive $H \mathbb{Z}$-modules is a map of graded pointed simplicial sets which is strictly compatible with the action of $H \mathbb{Z}$.

As shown in [62, Theorem B.1.3], the category $N v H \mathbb{Z}$-mod of naive $H \mathbb{Z}$-modules has a model structure in which the fibrations and the weak equivalences are created by the forgetful functor $U: N v H \mathbb{Z}$-mod $\rightarrow$ Sp from naive $H \mathbb{Z}$-modules to classical Bousfield-Friedlander spectra with the standard stable model structure of Bousfield and Friedlander [16, Theorem 2.3]. The model structure on $N v H \mathbb{Z}-$ mod is cellular and left proper, the latter by the same argument as in the proof of Theorem 3.16. One may also consider adjoints to $U$ as in the case of symmetric spectra (compare Theorem 3.16), but we will not need this in the sequel.

Theorem 3.23 (Schwede-Shipley) There is a zigzag of Quillen equivalences

$$
H \mathbb{Z}-\bmod \leftrightarrows N v H \mathbb{Z}-\bmod \rightleftarrows \mathrm{Ch}(\mathbf{A b})
$$

Proof See [62, Appendix B]. 


\section{Proof of Theorems 1.1 and 1.2}

Having established all necessary model structures and Quillen adjunctions in the previous section, we are now ready to prove Theorems 1.1 and 1.2. We will provide all details in the parts of the proof concerning classical spectra. When passing to diagram categories and motivic Bousfield localizations of those, we will provide details in the first couple of proofs, but allow ourselves to skip some of the by then familiar arguments in some of the later proofs. The reader interested in the classical case should simply think of the trivial site and ignore all localization functors with respect to the Nisnevich topology or to the affine line $\mathbf{A}^{1}$. The proof then becomes considerably shorter. In particular, most (but not all, see Proposition 3.9) model structures discussed in Section 3 are known in that case.

Let $\mathcal{M}$ be a simplicial monoidal model category and $T$ be a suitable pointed object in $\mathcal{M}$. We are mostly interested in two cases. Theorem 1.1 is about $\mathcal{M}=\Delta^{\mathrm{op}}$ Sets and $T=S^{1}$. Theorem 1.2 is about $\mathcal{M}$ being simplicial presheaves on $S m / S$ with the $\mathbf{A}^{1}$-local model structure recalled at the beginning of Section 3 , and $T=\mathbf{P}^{1}$, although throughout the proof for motivic symmetric spectra over $T=S^{1}$ will be considered as well. Note that the statements of the theorems are independent of the model structure one chooses, and by the previous section there is at least one for all the categories involved. Furthermore, we wish to emphasize once more that much of the proofs here, and in fact those of Section 3 as well, generalizes to other (simplicial) monoidal model categories. On the other hand, there are some key results which do not generalize. In particular, the theorems of [62] quoted below, and also Theorems 4.2 and 4.4 are really fundamental results specifically about classical Eilenberg Mac Lance spectra and delooping along the classical circle $S^{1}$, respectively. Results without $\mathcal{M}$ made explicit hold in the very general situation described above. We fix $N \in \operatorname{AbMon}(\mathcal{M})$ and $A \in \operatorname{AbMonSp}^{\Sigma, T}(\mathcal{M})$, and assume that $N$ is group-like, as defined below.

Putting everything together, we obtain the following diagram of categories and adjunctions, with the left adjoint displayed on top as usual. For simplicity, we only exhibit this diagram in the classical version, that is for $\mathcal{M}=\Delta^{\mathrm{op}}$ Sets. It generalizes to diagram categories, in particular with the site $\mathcal{C}=(\mathrm{Sm} / \mathrm{S})_{\mathrm{Nis}}$ as index category, and to various motivic localizations of those, as explained above and below. All categories are simplicial model categories, and all adjunctions are Quillen (one is even an actual equivalence of categories preserving the model structure). The global picture is that there are compatible forgetful functors $U$ from the left to the right column. The functor $V$ in the right column is defined in $[35,4.3]$ and extends to a functor $L$ in the left column by [62, B.1]. The functor $U$ in the top row is studied in Theorem 3.16. The 
upper right adjunction is a Quillen equivalence [35, Theorem 4.2.5] for $\mathcal{M}=\Delta^{\mathrm{op}}$ Sets (here Sp denotes Bousfield-Friedlander spectra [16]), and by [37, Theorem 4.40] for motivic symmetric $S^{1}$-spectra. The left column is explained at the end of the previous section. The dotted adjunction between $E_{\infty}-\operatorname{alg}\left(\Delta^{\mathrm{op}}\right.$ Sets $)$ and $\mathrm{Sp}$ is standard in other models for the stable homotopy category; see Lemma 4.1 below. The other dotted arrow to which it restricts is an equivalence of homotopy categories enriched over $\mathrm{Ho}\left(\Delta^{\mathrm{op}}\right.$ Sets $)$ which was proved by Ando, Blumberg, Gepner, Hopkins and Rezk in their [3, Theorem 3.45]; compare our Theorem 4.2 below. This is a variant of the famous recognition principle due to May [46] and Boardman-Vogt [13]. We write $E_{\infty}$ for the Barratt-Eccles operad $\mathcal{W}$ introduced in Definition 3.8. The precise meaning of the dotted arrows in our setting will be explained further below.

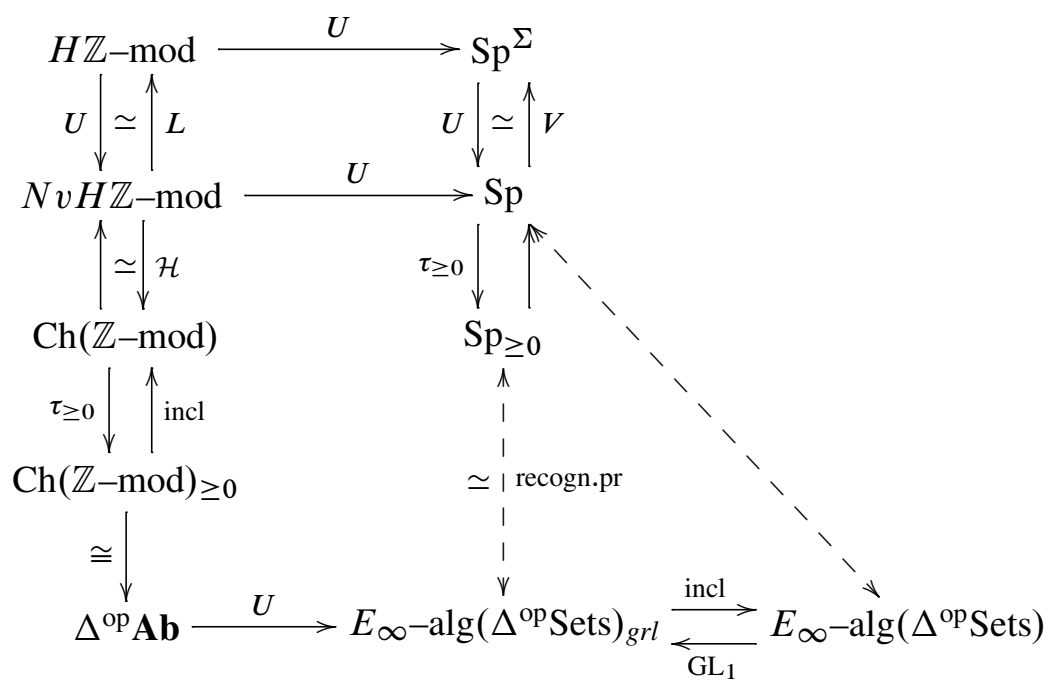

Lemma 4.1 In the world of Lewis-May-Steinberger spectra Sp [40], we have a Quillen adjunction of topological model categories

$$
\Sigma^{f}: E_{\infty}-\operatorname{alg}\left(\Delta^{\mathrm{op}} \mathrm{Sets}\right) \rightleftarrows \mathrm{Sp}: \Omega^{f}
$$

enriched over topological spaces.

Proof See [3, Lemma 3.43].

The construction of the functor $\Omega^{f}$ uses the linear isometries operad which is built in the definition of Lewis-May-Steinberger spectra, and thus is a key ingredient when proving the following theorem in their setting. 


\section{Theorem 4.2 There is an equivalence of homotopy categories}

$$
\operatorname{Ho}\left(E_{\infty}-\operatorname{alg}\left(\Delta^{\mathrm{op}} \mathrm{Sets}\right)_{g r l}\right) \simeq \operatorname{Ho}\left(\mathrm{Sp}_{\geq 0}\right),
$$

enriched over $\operatorname{Ho}\left(\Delta^{\mathrm{op}}\right.$ Sets $)$.

We will prove this theorem in our situation, that is for Bousfield-Friedlander spectra in simplicial sets $\mathrm{Sp}$ and its motivic generalizations. For this we will use a variant of Lemma 4.1 which we learned from Schwede, and the symmetric spectra variant of which will presumably be included in the final version of [60]. Namely, we consider the following zigzag diagram of Quillen adjunctions

$$
\mathrm{Sp} \rightleftarrows \mathrm{Sp}\left(E_{\infty}-\operatorname{alg}\left(\Delta^{\mathrm{op}} \mathrm{Sets}\right)\right) \leftrightarrows E_{\infty}-\operatorname{alg}\left(\Delta^{\mathrm{op}} \operatorname{Sets}\right)
$$

with left adjunctions displayed on top and $\operatorname{Sp}\left(E_{\infty}-\operatorname{alg}\left(\Delta^{\mathrm{op}} \operatorname{Sets}\right)\right)$ being the category of spectra with spaces and spectral structure maps all being $E_{\infty}$. The left free/forgetful adjunction creates a model structure on $\operatorname{Sp}\left(E_{\infty}-\operatorname{alg}\left(\Delta^{\text {op }} \operatorname{Sets}\right)\right)$ as usual, that is using the same arguments as for the existence of the stable model structure on $\mathrm{Sp}$ from $\Delta^{\mathrm{op}}$ Sets. (This also can be done in the motivic case below, starting with the model structure on $\left(E_{\infty}-\operatorname{alg}\left(\Delta^{\mathrm{op}} \operatorname{PrShv}(\mathcal{C})\right)\right.$ established in Theorem 3.12.) In the right Quillen adjunction, the functor $E v_{0}: \operatorname{Sp}\left(E_{\infty}-\operatorname{alg}\left(\Delta^{\mathrm{op}} \operatorname{Sets}\right)\right) \rightarrow E_{\infty}-\operatorname{alg}\left(\Delta^{\mathrm{op}} \operatorname{Sets}\right)$ is the usual evaluation at the 0 th space which is a right Quillen functor. However its left adjoint is not the naive $\Sigma^{\infty}$, but defined using the simplicial model structure on $E_{\infty}-\operatorname{alg}\left(\Delta^{\text {op }}\right.$ Sets) (which is induced by the Quillen adjunction with $\Delta^{\mathrm{op}}$ Sets) when defining the smash products with $S^{n}$ to define the level $n$-space of an object in $\operatorname{Sp}\left(E_{\infty}-\operatorname{alg}\left(\Delta^{\text {op }} \operatorname{Sets}\right)\right)$.

Now by the recognition principle, the left Quillen adjunction induces an equivalence of (enriched) homotopy categories, and hence (see eg [33, Proposition 1.3.13]) is an (enriched) Quillen equivalence. Moreover, the right Quillen adjunction induces an equivalence of (enriched) homotopy categories $\operatorname{Ho}\left(E_{\infty}-\operatorname{alg}\left(\Delta^{\mathrm{op}} \operatorname{Sets}\right)_{g r l}\right) \simeq$ $\operatorname{Ho}\left(\operatorname{Sp}\left(E_{\infty}-\operatorname{alg}\left(\Delta^{\mathrm{op}} \operatorname{Sets}\right)\right)_{\geq 0}\right)$. The latter equivalence can also be formulated as a Quillen equivalence, using suitable localizations of the above model structures on $E_{\infty}-\operatorname{alg}\left(\Delta^{\mathrm{op}} \operatorname{Sets}\right)$ and $\operatorname{Sp}\left(E_{\infty}-\mathrm{alg}\left(\Delta^{\mathrm{op}} \operatorname{Sets}\right)\right.$ as we now explain. (We do explain the localized model structure on $\operatorname{Sp}$ only, for $\operatorname{Sp}\left(E_{\infty}-\operatorname{alg}\left(\Delta^{\mathrm{op}} \operatorname{Sets}\right)\right)$, the arguments are exactly the same.) In modern language, see for example Hirschhorn [30, Section 5] or Smith [5, Section 5], these are examples of right Bousfield localizations, that is increasing the class of weak equivalences while keeping the same fibrations. I do not know of any published reference for the following proposition for sequential or symmetric spectra, but I learned that there is work in progress by Sagave and Schlichtkrull now available; see [57] — who apply similar techniques to study similar questions for $I$-spaces. One should also compare [52, Section 3.2] for a detailed discussion of how 
to lift the motivic Postnikov decomposition to the level of model structures using right Bousfield localizations, which contains precisely the arguments needed in our slightly easier case. Of course, the set $C_{\text {eff }}^{0}$ of [52] simply becomes the set of $S^{1}$-suspension spectra of the simplicial spheres.

Proposition 4.3 (i) The category $\mathrm{Sp}$ has a simplicial model structure with the same fibrations as the ones in [16, Theorem 2.3] and weak equivalences the $\pi_{n}$-isomorphisms for $n \geq 0$.

(ii) The category $E_{\infty}-\operatorname{alg}\left(\Delta^{\text {op }}\right.$ Sets) has a simplicial model structure with the same fibrations as above, that is fibrations on underlying simplicial sets, and a map being a weak equivalence if it is one after restricting to the invertible components.

Proof We apply the dual of Bousfield's Theorem [15, Theorems 9.3 and 9.7] to the Postnikov truncation $Q=\tau_{\geq 0}$ on $\mathrm{Sp}$ resp. to $Q=(-)^{\times}=$unital components on $E_{\infty}-\operatorname{alg}\left(\Delta^{\text {op }}\right.$ Sets) and the corresponding transformations $\alpha$. For (ii) the construction of $Q$ and $\alpha$ is obvious, and for (i) the reader may eg consult [60, Section III.5]. The category $\mathrm{Sp}$ is proper by [16, Theorem 2.3], and [15, 9.2, Properties (A1) and (A2)] are obviously satisfied. The dual of axiom (A3) follows as the hypothesis of [15] yield a homotopy pushout square and hence isomorphisms $0=Q \operatorname{cone}(h) \simeq Q \operatorname{cone}(k)$ as required. This finishes part (i). For part (ii), the category $E_{\infty}-\operatorname{alg}\left(\Delta^{\text {op }}\right.$ Sets) is right proper because the forgetful functor to the proper model category $\Delta^{\text {op }}$ Sets preserves limits, fibrations and weak equivalences. Showing that it is also left proper is a bit more subtle; see [65, Theorem 4] (or [21, Theorem 12.4.B]). The definition of left proper in loc. cit. coincides with the usual definition as all objects in $\Delta^{\text {op }}$ Sets are cofibrant. In order to apply the theorem of loc. cit. concerning left properness, we need to now that the operad in question is cofibrant for the model structure of loc. cit., which is created by the one of symmetric sequences. That one is equipped with the product model structure of equivariant simplicial sets which in turn is created by the one of $\Delta^{\text {op }}$ Sets forgetting the group action. This implies that the Barratt-Eccles operad $\mathcal{W}$ is $\Sigma$-cofibrant, which by definition means that its underlying symmetric sequence is cofibrant, as all $\Sigma_{n}$ act freely. Now we choose a cofibrant replacement $\mathcal{W}_{\text {cof }}$ of $\mathcal{W}$ (which itself is not cofibrant as Fresse kindly explained to me), which then in particular is also $\Sigma$-cofibrant (see eg [8, Proposition 4.3]). Finally, the model categories $\mathcal{W}$-alg

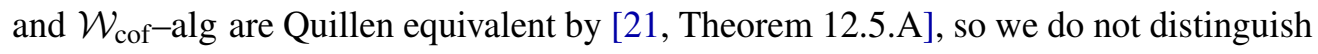
between them in our notation in the sequel. The dual statements of the conditions (A1), (A2) and (A3) of [15] are again easy to check.

Putting everything together and again suppressing the Quillen equivalence between Sp and $\operatorname{Sp}\left(E_{\infty}-\operatorname{alg}\left(\Delta^{\text {op }}\right.\right.$ Sets $\left.)\right)$ in our notation, we obtain the following square of Quillen 
adjunctions (model structures omitted from the notation). It corresponds to the lower right triangle in the large diagram of Quillen adjunctions above before Lemma 4.1.

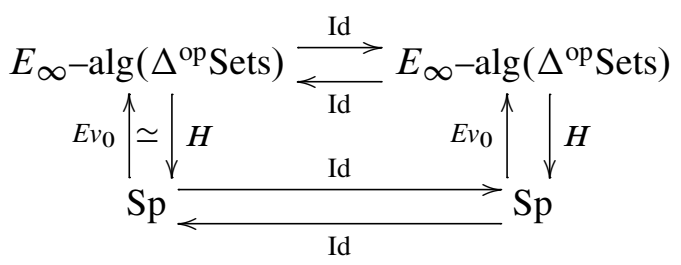

We claim that the left vertical pair is a Quillen equivalence. The horizontal equivalences are the right Bousfield localizations we just described. In more detail, the cofibrant objects in the left hand side model categories are the cofibrant objects with respect to the model structures on the right hand side which are moreover group-like $E_{\infty}$-spaces resp. (-1)-connected spectra. Hence the Quillen adjunction on the right hand side induces one on the left hand side. This is an example of [30, Theorem 3.3.20(2)(a)]: note that [30, Definition 8.5.11 (2)(a)] applied to right localization with respect to $\tau_{\geq 0}$ does not produce additional weak equivalences in $E_{\infty}$-algebras and therefore induces a Quillen adjunction between the lower left and the upper right corner in the above diagram. Composing this Quillen adjunction with the one on top leads to the one of the left hand side we are looking for. As we already pointed out above, this Quillen adjunction then induces an equivalence of homotopy categories by the recognition principle, hence it is a Quillen equivalence (see eg [33, Proposition 1.3.13]).

Recall that when writing $E_{\infty}-\operatorname{alg}\left(\Delta^{\text {op }} S e t s\right)_{g r l}$ resp. $\mathrm{Sp}_{\geq 0}$ in the large diagram above and further below, we really mean the model categories $E_{\infty}-\operatorname{alg}\left(\Delta^{\mathrm{op}} S e t s\right)$ resp. Sp with the right localized model structures established in Proposition 4.3. The total right derived functors of the right adjoint identity functors in the above square are precisely $\mathrm{GL}_{1}$ resp. the Postnikov functor $\tau_{\geq 0}$, thus justifying the labels on the arrows in the big diagram further above. This finishes our discussion of the proof of Theorem 4.2 and its refined formulation in the language of model categories.

Again, all above Quillen adjunctions and equivalences in the above diagram generalize to the motivic situation using always the same kind of arguments involving left Bousfield localizations of diagram categories.

Theorem 4.4 The above Quillen adjunctions induce Quillen adjunctions for the corresponding motivic categories, which then induce an equivalence of homotopy categories

$$
\operatorname{Ho}\left(E_{\infty}-\operatorname{alg}\left(\Delta^{\mathrm{op}} \operatorname{PrShv}(\mathcal{C})\right)_{g r l}\right) \simeq \operatorname{Ho}\left(\operatorname{Sp}^{S^{1}}(\mathcal{C})_{\geq 0}\right)
$$

enriched over $\operatorname{Ho}\left(\Delta^{\mathrm{op}} \operatorname{Sets}\right)$ and even over $\operatorname{Ho}\left(\Delta^{\mathrm{op}} \operatorname{PrShv}(\mathcal{C})\right)$. 
Proof The above Quillen adjunction between $E_{\infty}$-spaces and spectra generalizes to one between global model structures on diagram categories (see for example Hirschhorn [30, Theorems 11.6.1 and 11.6.5]). We claim that this one then induces a Quillen adjunction after left Bousfield localization on both sides with respect to the Nisnevich topology and to $\mathbf{A}^{1}=\mathbf{A}_{S}^{1} \rightarrow S$ by standard arguments, that is by [30, Theorem 3.3.20(1)(a)]. On $\left(E_{\infty}-\operatorname{alg}\left(\Delta^{\mathrm{op}} \operatorname{PrShv}(\mathcal{C})\right)\right)$, this is precisely the model structure established in Theorem 3.12. Indeed, looking at the fibrant replacement functors discussed there, we see that we obtain the correct $\mathbf{L F C}$ in the notation of [30]. As before, we then wish to apply a suitable right Bousfield localization to these left localized motivic model structures, thus obtaining the homotopy categories of connected motivic $S^{1}$-spectra and grouplike motivic $E_{\infty}$-spaces using the (diagram versions of) the right Bousfield localizations considered in Proposition 4.3. To see that the right localization of the $\mathbf{A}^{1}$-local structures on motivic $E_{\infty}$-spaces exists, we may apply the dual of [15] as before. The arguments above imply that motivic $E_{\infty}$-spaces are cellular and left proper. To see that they are right proper, recall that motivic spaces are right proper and the $\mathbf{A}^{1}$-local model structure on $E_{\infty}$-spaces is created by the forgetful functor which preserves pullbacks. Then one checks the dual of the remaining hypotheses of [15] with respect to $Q=(-)^{\times}$. Concerning the right localization of motivic $S^{1}$-spectra, it is more convenient to apply [30, Theorem 5.1.1] rather than [15]. That is, we proceed as Pelaez does in [52, Section 3.2]. Recall that motivic $S^{1}$-spectra are cellular and proper by Hovey and Jardine, that is (the sequential spectra version of) Theorem 3.1. From the (sequential spectra version of) [52, Proposition 3.2.4] it easily follows that the right Quillen functor $E v_{0}$ with respect to the motivic model structures remains right Quillen when applied to the right localizations we just described. Since it induces an equivalence of homotopy categories, then it is a Quillen equivalence as claimed.

As Pelaez explained to me, Morel's connectivity result (which is valid only for $S=\operatorname{Spec}(k)$ ) really is stronger than what we have used here. It can of course not be recovered using only the above techniques.

Now we are ready for the proof of the main theorems. We write down a chain of natural weak equivalences of simplicial sets, so applying $\pi_{0}$ yields Theorems 1.1 and 1.2. To simplify notation, we drop all base points in the sequel.

Let $\mathcal{M}$ be one of the two monoidal model categories we are interested in; see the beginning of this section. We have

$$
\operatorname{Rmap}_{\left.\operatorname{AbMon}\left(\mathrm{Sp}^{\Sigma, T}(\mathcal{M})\right)\right)}\left(\Sigma_{T}^{\infty} N, A\right) \simeq \operatorname{Rmap}_{\left.E_{\infty}\left(\mathrm{Sp}^{\Sigma, T}(\mathcal{M})\right)\right)}\left(\Sigma_{T}^{\infty} N, A\right),
$$

using Theorem 3.10 and flat positive stable model structures. Now the identity is a Quillen equivalence between the positive and the nonpositive (see Theorem 3.6 and 
Proposition 3.9) model structure, so we may switch to the latter on $\left.E_{\infty}\left(\mathrm{Sp}^{\Sigma, T}(\mathcal{M})\right)\right)$. Then using Corollary 3.14, we have

$$
\simeq \operatorname{Rmap}_{E_{\infty}(\mathcal{M})}\left(N, E v_{0}(A)\right) .
$$

As $N$ is an abelian group by assumption, hence grouplike, we have

$$
\simeq \operatorname{Rmap}_{E_{\infty}(\mathcal{M})_{g r l}}\left(N, \operatorname{GL}_{1}\left(E v_{0}(A)\right)\right)
$$

by Proposition 4.3 above. Recall the meaning of the heuristic notation $E_{\infty}(\mathcal{M})_{g r l}$ and $\mathrm{GL}_{1}$ as introduced immediately after loc. cit., it would be more accurate to say that " $N$ is cofibrant in the right localized model structure of Proposition 4.3". The chain of weak equivalences continues with

$$
\simeq \operatorname{Rmap}_{\mathrm{Sp}_{\geq 0}}\left(H N, g l_{1}(A)\right),
$$

using Theorem 4.2 resp. Theorem 4.4 and its proof, which defines $H N$ and $g l_{1}(A)$. Observe that $\mathrm{GL}_{1} \circ E v_{0}=E v_{0} \circ g l_{1}$, and moreover $g l_{1}$ allows a model-theoretic description as right Quillen adjoint similar to $\mathrm{GL}_{1}$. As before, we have suppressed the left hand side Quillen equivalence in the zigzag of the Quillen adjunctions after Theorem 4.2 from our notation. Recall also that we are dealing with $S^{1}$-spectra and the usual Eilenberg-Mac Lane spaces here. The next weak equivalence

$$
\simeq \operatorname{Rmap}_{N v H \mathbb{Z}-\operatorname{Mod}\left(\mathrm{Sp}_{\geq 0}(\mathcal{M})\right)}\left(H N, \operatorname{Rmap}_{\mathrm{Sp}_{\geq 0}(\mathcal{M})}\left(H \mathbb{Z}, g l_{1}(A)\right)\right)
$$

is just a formal adjunction; see Theorem 3.16 which allows a variant for "naive" $H \mathbb{Z}-$ modules. Note in particular that $H N$ is a module over $H \mathbb{Z}$. Then

$$
\simeq \operatorname{Rmap}_{A b(\mathcal{M})}\left(N, \operatorname{Rmap}_{E_{\infty}(\mathcal{M})_{g r l}}\left(\mathbb{Z}, \operatorname{GL}_{1}\left(E v_{0}(A)\right)\right)\right),
$$

using [62, Theorem 3.23] of Schwede-Shipley resp. its motivic generalization and Theorems 3.20 and 3.21. Note that this is compatible with Theorem 4.2 by the large commutative diagram above. In particular, $\operatorname{Rmap}_{E_{\infty}(\mathcal{M})_{g r l}}\left(\mathbb{Z}, \mathrm{GL}_{1}\left(E v_{0}(A)\right)\right)$ no longer denotes a (presheaf of) $H \mathbb{Z}$-module(s), but the corresponding (presheaf of) simplicial abelian group(s). Observe that the model structures on $\operatorname{Ab}(\mathcal{M})$ and $\operatorname{AbMon}(\mathcal{M})$ are compatible since both are created via the forgetful functor to $\mathcal{M}$. Then

$$
\begin{aligned}
& \simeq \operatorname{Rmap}_{\operatorname{AbMon}(\mathcal{M})}\left(N, \operatorname{Rmap}_{E_{\infty}(\mathcal{M})}\left(\mathbb{Z}, \operatorname{GL}_{1}\left(E v_{0}(A)\right)\right)\right) \\
& \simeq \operatorname{Rmap}_{A b M o n(\mathcal{M})}\left(N, \operatorname{Rmap}_{E_{\infty}(\mathcal{M})}\left(\mathbb{Z}, E v_{0}(A)\right)\right),
\end{aligned}
$$

as $\mathbb{Z}$ is grouplike again by Proposition 4.3 above. Then

$$
\simeq \operatorname{Rmap}_{\operatorname{AbMon}(\mathcal{M})}\left(N, \operatorname{Rmap}_{E_{\infty}\left(\mathrm{Sp}^{\Sigma, T}(\mathcal{M})\right)}\left(\Sigma_{T}^{\infty} \mathbb{Z}, A\right)\right),
$$


and finally proceeding as above,

$$
\simeq \operatorname{Rmap}_{\operatorname{AbMon}(\mathcal{M})}\left(N, \operatorname{Rmap}_{\operatorname{AbMon}\left(\operatorname{Sp}^{\Sigma, T}(\mathcal{M})\right)}\left(\Sigma_{T}^{\infty} \mathbb{Z}, A\right)\right) .
$$

Note that the above chain of weak equivalences really arises from "zigzags", as various of the enriched Quillen equivalences in the above argument go in the "wrong" direction. To start with, the identity is a left Quillen adjoint from the positive to the absolute model structure on symmetric spectra and algebras over those. When considering derived mapping spaces, we must choose a cofibrant and a fibrant replacement functor for both model structures. In this situation, we may simply choose the cofibrant replacement functor with respect to positive model structure (which then also is one for the absolute model structure) and the fibrant replacement functor with respect to the absolute model structure (which then is also one for the positive model structure). These choices show that the chain of weak equivalences leading to the Main Theorems really can be chosen to be one with is natural both in $N$ and $A$. Another such zigzag is hidden in the recognition principle, and still another one in the motivic generalization of [62].

Putting everything together, we therefore have a natural weak equivalence of derived simplicial mapping spaces

$$
\begin{aligned}
\operatorname{Rmap}_{\left.\operatorname{AbMon}\left(\operatorname{Sp}^{\Sigma, T}(\mathcal{M})\right)\right)}\left(\Sigma_{T}^{\infty}\right. & N, A) \\
& \simeq \operatorname{Rmap}_{\operatorname{AbMon}(\mathcal{M})}\left(N, \operatorname{Rmap}_{\operatorname{AbMon}\left(\operatorname{Sp}^{\Sigma, T}(\mathcal{M})\right)}\left(\Sigma_{T}^{\infty} \mathbb{Z}, A\right)\right),
\end{aligned}
$$

which after applying $\pi_{0}$ finishes the proof of Theorems 1.1 and 1.2.

\section{Motivic preorientations and orientations of the derived multiplicative group}

In this section, we explain how the Theorems 1.1 and 1.2 lead to the results about orientations and $K$-theory stated in the introduction.

Theorem 1.2 applies to $N=\bar{W}\left(\mathrm{GL}_{1}\right) \simeq \mathbf{P}^{\infty} \in \Delta^{\mathrm{op}} \operatorname{PrShv}(\mathcal{C})$. Here, $\bar{W}(\cdot)$ is a specific model for the classifying space of a simplicial group; see for example Goerss and Jardine [24, Chapter V, 4]. It is easy to see that $\bar{W}(\cdot)$ sends commutative simplicial abelian groups to commutative monoids in $\Delta^{\mathrm{op}} \operatorname{PrShv}(\mathcal{C})$. The equivalence $\bar{W}\left(\mathrm{GL}_{1}\right) \simeq \mathbf{P}^{\infty}$ is a special case of [48, Proposition 3.7]. Beware of the difference between $\mathrm{GL}_{1}$ and the $\mathbf{G}_{m}$ above. In Theorem 1.1, the same argument applies to the topological group $S^{1}=U(1)$ with classifying space $\mathrm{CP}^{\infty}$.

We do not give a definition of the notion of a derived group scheme in $\operatorname{AbMonSp}^{\Sigma, T}(\mathcal{C})$ here. Compare [42, Section 3] for a motivation of the following definition, at least for the trivial site. 
Definition 5.1 A preorientation on a derived group scheme $G$ over a motivic symmetric spectrum $A$ is an element in $\operatorname{Hom}_{\mathrm{AbMon} \Delta^{\mathrm{opPrShv}}(\mathcal{C})}\left(\bar{W}\left(\mathrm{GL}_{1}\right), G(A)\right)$.

Note that this definition is related, but not equivalent to more classical notions of orientations as eg in Adams' book [2]. (Namely, in contrast to [2], we consider strict monoid homomorphisms to the infinite dimensional projective space, and also we do consider such maps only up to homotopy.) However, by [42] it is the "correct" definition in order to obtain the right definition of tmf, and in the height 1 case to obtain $K O$.

One easily checks that for any simplicial abelian monoid, the associated suspension spectrum is a commutative motivic ring spectrum (compare [60, Example I.2.32] and Lemma 2.3). Theorem 1.2 may thus be rephrased as follows in the special case of $\mathbf{P}^{\infty}$, where we write $S[-]$ for $\Sigma_{T}^{\infty}(-)$ following Lurie's notation (as introduced in the beginning of Section 2 already).

Theorem 5.2 There is a bijection between preorientations of $\mathbf{G}_{m}$ over $A$ and $\operatorname{Hom}_{\mathrm{Ho}\left(\operatorname{AbMonSp}^{\Sigma, T}(\mathcal{C})\right)}\left(S\left[\bar{W}\left(\mathrm{GL}_{1}\right)\right], A\right)$. In other words, $S\left[\bar{W}\left(\mathrm{GL}_{1}\right)\right]$ classifies preorientations of the derived multiplicative group.

Lurie also gives a definition of an orientation; see [42]. We do not suggest a motivic generalization of this definition in general, either. However, any reasonable generalization of the notion of an orientation from the classical to the motivic case will certainly imply a bijection between the set of orientations on $\mathbf{G}_{m}$ over $A$ and the set $\operatorname{Hom}_{\mathrm{Ho}\left(\mathrm{AbMonSp}^{\Sigma, T}(\mathcal{C})\right)}\left(S\left[\bar{W}\left(\mathrm{GL}_{1}\right)\right]\left[\beta^{-1}\right], A\right)$ for a certain lift of the motivic Bott element $\beta$ (see below). In other words, $S\left[\bar{W}\left(\mathrm{GL}_{1}\right)\right]\left[\beta^{-1}\right]$ will classify orientations of the derived multiplicative group.

By recent work of Spitzweck-Østvær [66] and independently of Gepner-Snaith [22], we have the following algebraic version of Snaith's theorem.

Theorem 5.3 (Spitzweck-Østvær and Gepner-Snaith) There is an isomorphism of commutative monoids in $S H(S)$ between the underlying motivic spectrum of $S\left[\bar{W}\left(\mathrm{GL}_{1}\right)\right]\left[\beta^{-1}\right]$ and Voevodsky's motivic spectrum representing algebraic $K$-theory (see [68, Section 6.2]) where $\beta \in \pi_{2,1}\left(B G L_{1}\right)$ is a lift of the motivic Bott element.

In light of this result, our Theorem 5.2 above may be rephrased by saying that algebraic $K$-theory classifies orientations of the derived multiplicative group. More precisely, one must either assume $S$ regular here or work with Weibel's homotopy invariant algebraic $K$-theory [70] for nonregular base schemes.

The classical Snaith theorem [64] together with some considerations about suspension spectra and suitable localizations of those being semistable symmetric ring spectra 
leads to a description of topological $K$-theory as a strictly commutative ring spectrum, that is an abelian monoid in symmetric spectra. More precisely, suspension spectra are "semistable" in the sense of [60, Theorem I.4.42] by [60, Example I.4.46]. It follows that $S\left[\bar{W}\left(\mathrm{GL}_{1}\right)\right]\left[\beta^{-1}\right]$ is again a symmetric spectrum by [60, Corollary I.4.67], hence complex topological $K$-theory is represented by a strictly commutative ring spectrum in $\mathrm{Sp}^{\Sigma}$. This argument generalizes to the motivic situation.

Proposition 5.4 (Röndigs, Spitzweck, Østvær) The object $S\left[\bar{W}\left(\mathrm{GL}_{1}\right)\right]\left[\beta^{-1}\right]$ is a commutative monoid in $\mathrm{Sp}^{\Sigma, T}(\mathcal{C})$.

Proof See Röndigs, Spitzweck and Østvær [56].

Returning again to the classical case, the fact that $K_{\text {top }}^{h \mathbb{Z} / 2} \simeq K O_{\text {top implies that } K O}$ classifies all oriented derived multiplicative groups; see [42, Remark 3.12] for details. A similar statement for algebraic and hermitian $K$-theory, namely $K_{\text {alg }}^{h \mathbb{Z} / 2} \simeq K O_{\text {alg }}$ was conjectured to hold for arbitrary rings with 2 invertible at least after a suitable completion; see Williams [71, 3.4.2]. This conjecture has been proved in many cases; see Kobal [39], Berrick, Karoubi and Østvær [10] and more recently Hu, Kriz and Ormsby [36], Berrick, Karoubi, Schlichting and Østvær [11], but in general it is wrong as shown in [10].

Finally, let us mention that there are of course many examples of abelian monoids in symmetric $T$-spectra. Suspension spectra of abelian monoids, eg of algebraic groups or abelian varieties, are obvious examples. Another example is Voevodsky's algebraic cobordism spectrum MGL, as explained by Panin and Yagunov in [51, Section 6.5] and Panin, Pimenov and Röndigs [50, Section 2.1]. The techniques of Schlichtkrull [58] then yield many more examples, as the proof of Theorem 1.1 of loc. cit. carries over to the motivic Thom spectrum, and hence applies to $\mathcal{I U} / B G L$ with $\mathcal{U}$ being the category of motivic spaces, that is simplicial presheaves. Moreover, one may try to use the isomorphism $\mathbf{A}^{n}-0 \simeq S^{n-1} \wedge \mathbf{G}_{m}^{n}$ to extend the picture to a motivic version of generalized Thom spectra with respect to a motivic version of $B F$, that is with self maps on $T^{n}$. We might pursue this topic in some other article.

\section{References}

[1] J Adámek, J Rosický, Locally presentable and accessible categories, London Mathematical Society Lecture Note Series 189, Cambridge Univ. Press (1994) MR1294136

[2] J F Adams, Stable homotopy and generalised homology, Chicago Lectures in Mathematics, University of Chicago Press (1974) MR0402720 
[3] M Ando, A Blumberg, D Gepner, M Hopkins, C Rezk, Units of ring spectra and Thom spectra arXiv:0810.4535

[4] M G Barratt, P J Eccles, $\Gamma^{+}$-structures, I: A free group functor for stable homotopy theory, Topology 13 (1974) 25-45 MR0348737

[5] C Barwick, On left and right model categories and left and right Bousfield localizations, Homology, Homotopy Appl. 12 (2010) 245-320 MR2771591

[6] M Behrens, Notes on the construction of tmf, preprint (2009) Available at http:// www-math.mit.edu/ mbehrens/papers/index.html

[7] C Berger, B Fresse, Combinatorial operad actions on cochains, Math. Proc. Cambridge Philos. Soc. 137 (2004) 135-174 MR2075046

[8] C Berger, I Moerdijk, Axiomatic homotopy theory for operads, Comment. Math. Helv. 78 (2003) 805-831 MR2016697

[9] J E Bergner, A survey of ( $\infty, 1)$-categories, from: "Towards higher categories", (J C Baez, J P May, editors), IMA Vol. Math. Appl. 152, Springer, New York (2010) 69-83 MR2664620

[10] A J Berrick, M Karoubi, PA Østvær, Hermitian $K$-theory and 2-regularity for totally real number fields, Math. Ann. 349 (2011) 117-159 MR2753799

[11] A J Berrick, M Karoubi, M Schlichting, PA Østvær, The homotopy fixed point theorem and the Quillen-Lichtenbaum conjecture in hermitian $K$-theory, preprint (2011) Available at http://www.math.uiuc.edu/K-theory/

[12] B A Blander, Local projective model structures on simplicial presheaves, $K$-Theory 24 (2001) 283-301 MR1876801

[13] J M Boardman, R M Vogt, Homotopy invariant algebraic structures on topological spaces, Lecture Notes in Mathematics 347, Springer, Berlin (1973) MR0420609

[14] F Borceux, Handbook of categorical algebra, 2, Encyclopedia of Mathematics and its Applications 51, Cambridge Univ. Press (1994) MR1313497

[15] A K Bousfield, On the telescopic homotopy theory of spaces, Trans. Amer. Math. Soc. 353 (2001) 2391-2426 MR1814075

[16] A K Bousfield, E M Friedlander, Homotopy theory of $\Gamma$-spaces, spectra, and bisimplicial sets, from: "Geometric applications of homotopy theory", Lecture Notes in Math. 658, Springer, Berlin (1978) 80-130 MR513569

[17] B I Dundas, O Röndigs, P A Østvær, Motivic functors, Doc. Math. 8 (2003) 489-525 MR2029171

[18] W G Dwyer, D M Kan, Singular functors and realization functors, Nederl. Akad. Wetensch. Indag. Math. 46 (1984) 147-153 MR749528

[19] A D Elmendorf, I Kriz, MA Mandell, J P May, Rings, modules, and algebras in stable homotopy theory, Mathematical Surveys and Monographs 47, American Mathematical Society (1997) MR1417719 
[20] A D Elmendorf, M A Mandell, Rings, modules, and algebras in infinite loop space theory, Adv. Math. 205 (2006) 163-228 MR2254311

[21] B Fresse, Modules over operads and functors, Lecture Notes in Mathematics 1967, Springer, Berlin (2009) MR2494775

[22] D Gepner, V Snaith, On the motivic spectra representing algebraic cobordism and algebraic K-theory, Doc. Math. 14 (2009) 359-396 MR2540697

[23] P G Goerss, M J Hopkins, Moduli spaces of commutative ring spectra, from: "Structured ring spectra", (A Baker, B Richter, editors), London Math. Soc. Lecture Note Ser. 315, Cambridge Univ. Press (2004) 151-200 MR2125040

[24] P G Goerss, J F Jardine, Simplicial homotopy theory, Progress in Mathematics 174, Birkhäuser, Basel (1999) MR1711612

[25] S Gorchinskiy, V Guletski, Symmetric powers in stable homotopy categories arXiv: 0907.0730

[26] J E Harper, Homotopy theory of modules over operads in symmetric spectra, Algebr. Geom. Topol. 9 (2009) 1637-1680 MR2539191

[27] J E Harper, Homotopy theory of modules over operads and non- $\Sigma$ operads in monoidal model categories, J. Pure Appl. Algebra 214 (2010) 1407-1434 MR2593672

[28] A Heller, Homotopy theories, Mem. Amer. Math. Soc. 71 (1988) vi+78 MR920963

[29] PS Hirschhorn, Letter to Paul Coerss (1996)

[30] PS Hirschhorn, Model categories and their localizations, Mathematical Surveys and Monographs 99, American Mathematical Society (2003) MR1944041

[31] J Hornbostel, Localizations in motivic homotopy theory, Math. Proc. Cambridge Philos. Soc. 140 (2006) 95-114 MR2197578

[32] M Hovey, Monoidal model categories arXiv:math/9803002

[33] M Hovey, Model categories, Mathematical Surveys and Monographs 63, American Mathematical Society (1999) MR1650134

[34] M Hovey, Spectra and symmetric spectra in general model categories, J. Pure Appl. Algebra 165 (2001) 63-127 MR1860878

[35] M Hovey, B Shipley, J Smith, Symmetric spectra, J. Amer. Math. Soc. 13 (2000) 149-208 MR1695653

[36] P Hu, I Kriz, K Ormsby, The homotopy limit problem for Hermitian K-theory, equivariant motivic homotopy theory and motivic real cobordism, Adv. Math. 228 (2011) 434-480 MR2822236

[37] J F Jardine, Motivic symmetric spectra, Doc. Math. 5 (2000) 445-553 MR1787949

[38] J F Jardine, Presheaves of spectra, Lectures at the Fields Institute (2007) Available at http://www. math. uwo.ca/ jardine/papers/Fields-02.pdf

[39] D Kobal, The Karoubi tower and $K$-theory invariants of Hermitian forms, $K$-Theory 17 (1999) 141-150 MR1696429 
[40] L G Lewis, Jr, J P May, M Steinberger, J E McClure, Equivariant stable homotopy theory, Lecture Notes in Mathematics 1213, Springer, Berlin (1986) MR866482

[41] J Lurie, Higher topos theory, Annals of Mathematics Studies 170, Princeton Univ. Press (2009) MR2522659

[42] J Lurie, A survey of elliptic cohomology, from: "Algebraic topology”, Abel Symp. 4, Springer, Berlin (2009) 219-277 MR2597740

[43] J Lurie, Higher algebra (2012) Available at http://www.math.harvard.edu/ lurie

[44] S Mac Lane, Categories for the working mathematician, 2nd edition, Graduate Texts in Mathematics 5, Springer, New York (1998) MR1712872

[45] M A Mandell, J P May, S Schwede, B Shipley, Model categories of diagram spectra, Proc. London Math. Soc. 82 (2001) 441-512 MR1806878

[46] J P May, The geometry of iterated loop spaces, Lectures Notes in Mathematics 271, Springer, Berlin (1972) MR0420610

[47] F Morel, The stable $\mathbb{A}^{1}$-connectivity theorems, $K$-Th. 35 (2005) 1-68 MR2240215

[48] F Morel, V Voevodsky, $\mathbb{A}^{1}$-homotopy theory of schemes, Inst. Hautes Études Sci. Publ. Math. (1999) 45-143 MR1813224

[49] N Naumann, M Spitzweck, P A Østvær, Motivic Landweber exactness, Doc. Math. 14 (2009) 551-593 MR2565902

[50] I Panin, K Pimenov, O Röndigs, A universality theorem for Voevodsky's algebraic cobordism spectrum, Homology, Homotopy Appl. 10 (2008) 211-226 MR2475610

[51] I Panin, S Yagunov, Rigidity for orientable functors, J. Pure Appl. Algebra 172 (2002) 49-77 MR1904229

[52] P Pelaez, Multiplicative properties of the slice filtration, Astérisque 335 (2011) MR2807904

[53] D G Quillen, Homotopical algebra, Lecture Notes in Mathematics 43, Springer, Berlin (1967) MR0223432

[54] C W Rezk, Spaces of algebra structures and cohomology of operads, PhD Thesis, Massachusetts Institute of Technology (1996)

[55] M Robalo, Noncommutative motives I: From commutative to noncommutative motives arXiv: 1206.3645

[56] O Röndigs, M Spitzweck, P A Østvær, Motivic strict ring models for $K$-theory, Proc. Amer. Math. Soc. 138 (2010) 3509-3520 MR2661551

[57] S Sagave, C Schlichtkrull, Diagram spaces and symmetric spectra, Adv. Math. 231 (2012) 2116-2193 MR2964635

[58] C Schlichtkrull, Thom spectra that are symmetric spectra, Doc. Math. 14 (2009) 699-748 MR2578805

[59] S Schwede, Stable homotopical algebra and $\Gamma$-spaces, Math. Proc. Cambridge Philos. Soc. 126 (1999) 329-356 MR1670249 
[60] S Schwede, An untitled book project about symmetric spectra, Preliminary version of a book (2007) Available at http://www.math.uni-bonn.de/people/schwede/

[61] S Schwede, B E Shipley, Algebras and modules in monoidal model categories, Proc. London Math. Soc. 80 (2000) 491-511 MR1734325

[62] S Schwede, B E Shipley, Stable model categories are categories of modules, Topology 42 (2003) 103-153 MR1928647

[63] B E Shipley, A convenient model category for commutative ring spectra, from: "Homotopy theory: relations with algebraic geometry, group cohomology, and algebraic $K$-theory", (P Goerss, S Priddy, editors), Contemp. Math. 346, Amer. Math. Soc. (2004) 473-483 MR2066511

[64] V Snaith, Localized stable homotopy of some classifying spaces, Math. Proc. Cambridge Philos. Soc. 89 (1981) 325-330 MR600247

[65] M Spitzweck, Operads, algebras and modules in general model categories arXiv: math/0101102

[66] M Spitzweck, P A Østvær, The Bott inverted infinite projective space is homotopy algebraic K-theory, Bull. Lond. Math. Soc. 41 (2009) 281-292 MR2496504

[67] B Toën, G Vezzosi, Homotopical algebraic geometry, II: Geometric stacks and applications, Mem. Amer. Math. Soc. 193 (2008) x+224 MR2394633

[68] V Voevodsky, $\mathbb{A}^{1}$-homotopy theory, from: "Proceedings of the International Congress of Mathematicians, Vol. I”, Extra Vol. I (1998) 579-604 MR1648048

[69] V Voevodsky, Open problems in the motivic stable homotopy theory. I, from: "Motives, polylogarithms and Hodge theory, Part I", (F Bogomolov, L Katzarkov, editors), Int. Press Lect. Ser. 3, Int. Press, Somerville, MA (2002) 3-34 MR1977582

[70] CA Weibel, Homotopy algebraic $K$-theory, from: "Algebraic $K$-theory and algebraic number theory”, (M R Stein, R K Dennis, editors), Contemp. Math. 83, Amer. Math. Soc. (1989) 461-488 MR991991

[71] B Williams, Quadratic $K$-theory and geometric topology, from: "Handbook of $K-$ theory, Vol. 1, 2”, (E M Friedlander, D R Grayson, editors), Springer, Berlin (2005) 611-651 MR2181831

Fachbereich C, Mathematik und Informatik, Bergische Universität Wuppertal

Gaußstrasse 20, D-42119 Wuppertal, Germany

hornbostel@math.uni-wuppertal.de.

http://www2 . math. uni-wuppertal.de/ hornbost/

Received: 22 March 2012 Revised: 2 April 2013 